\title{
Pacific
}

Journal of

Mathematics

\section{ON RECURRENCE OVER SUBSETS AND WEAK MIXING}

JiAn Li, PIOTR OPROCha AND GuOHUA Zhang 


\title{
ON RECURRENCE OVER SUBSETS AND WEAK MIXING
}

\author{
JiAn Li, Piotr Oprocha AND GUOHUA ZHANG
}

\begin{abstract}
We study properties of weakly mixing sets (of order $n$ ) in relation to proximality, sensitivity, scrambled tuples, Xiong chaotic sets and independent sets. Our main emphasis is on the structure of the set of transfer times $N(U \cap A, V)$ between open sets $U$ and $V$, both intersecting a weakly mixing set $A$. We find several conditions on properties of the set $A$ that are equivalent to weak mixing.

We also prove that on topological graphs weakly mixing sets of order 2 can be approximated arbitrarily closely by a weakly mixing set of all orders. This property is known to hold on the unit interval but is not true in general (there are systems with weakly mixing sets of order $n$ but not $n+1$ ).
\end{abstract}

\section{Introduction}

This paper is a continuation of the previous papers by Oprocha and Zhang on local aspects of topological weak mixing $[2011 ; 2012 ; 2013 ; 2014]$ in dynamical systems $(X, f)$, that is, continuous maps $f: X \rightarrow X$ acting on compact metric spaces. When defining recurrent properties of dynamical systems, it is convenient to analyze properties of transfer times between sets, expressed in terms of the set

$$
N(U, V)=\left\{n \in \mathbb{N}: f^{n}(U) \cap V \neq \varnothing\right\},
$$

where $U$ and $V$ are nonempty open subsets of $X$. For example, $(X, f)$ is transitive if $N(U, V)$ is nonempty for any choice of two nonempty open sets. As mentioned before, the main concept in this paper is topological weak mixing (in fact, its local versions), which is usually defined as transitivity of $(X \times X, f \times f)$. In other words, $(X, f)$ is weakly mixing if $N\left(U_{1}, V_{1}\right) \cap N\left(U_{2}, V_{2}\right) \neq \varnothing$ for any choice of four nonempty open sets $U_{1}, U_{2}, V_{1}, V_{2} \subset X$. It was shown by Furstenberg [1967] that if $(X, f)$ is weakly mixing then for every $n \geq 2$ and any nonempty open sets $U_{1}, U_{2}, \ldots, U_{n}, V_{1}, V_{2}, \ldots, V_{n} \subset X$ we have

$$
N\left(U_{1}, V_{1}\right) \cap N\left(U_{2}, V_{2}\right) \cap \cdots \cap N\left(U_{n}, V_{n}\right) \neq \varnothing .
$$

Guohua Zhang is the corresponding author.

MSC2010: primary 37B05; secondary 37B40, 37E05, 37E25.

Keywords: weakly mixing sets, Xiong chaotic sets, proximality, scrambled tuples, independent sets, topological graphs. 
Note that weak mixing can be regarded as a 'global' property, while topological entropy is a 'local' one since it can be supported on a small set in the space (e.g., a nowhere dense attractor). It is also not hard to see that in general there cannot be any implication between weak mixing and positive topological entropy. Therefore an appropriate 'local' version of weak mixing is needed. Such a concept was introduced in [Blanchard and Huang 2008]. Strictly speaking, a nontrivial closed set $A \subset X$ (i.e., not a singleton) is weakly mixing if for every $n \geq 2$ and any nonempty open sets $U_{1}, U_{2}, \ldots, U_{n}, V_{1}, V_{2}, \ldots, V_{n} \subset X$ intersecting $A$ (i.e., $U_{i} \cap A \neq \varnothing$ and $V_{i} \cap A \neq \varnothing$ for each $\left.i=1,2, \ldots, n\right)$ we have $N\left(U_{1} \cap A, V_{1}\right) \cap N\left(U_{2} \cap A, V_{2}\right) \cap$ $\cdots \cap N\left(U_{n} \cap A, V_{n}\right) \neq \varnothing$. As we can see, the above definition is consistent with the definition of a weakly mixing map and, more importantly, it is proved in [Blanchard and Huang 2008] that every dynamical system with positive topological entropy contains many Cantor weakly mixing sets.

Similarly, for a fixed integer $n \geq 2$, we say that a nontrivial closed subset $A$ of $X$ is weakly mixing of order $n$ if for any nonempty open subsets $U_{1}, U_{2}, \ldots, U_{n}$, $V_{1}, V_{2}, \ldots, V_{n}$ of $X$ intersecting $A$, we have $N\left(U_{1} \cap A, V_{1}\right) \cap N\left(U_{2} \cap A, V_{2}\right) \cap \cdots \cap$ $N\left(U_{n} \cap A, V_{n}\right) \neq \varnothing$. Unfortunately, the analog of Furstenberg's theorem cannot be proved here. Namely, it is proved in [Oprocha and Zhang 2011; 2014] that for every $n \geq 2$ there exists a dynamical system which contains weakly mixing sets of order $n$ but no weakly mixing sets of order $n+1$.

Since Furstenberg's theorem does not work for weakly mixing sets of order $n$, it is natural to ask which criteria for weak mixing (i.e., equivalent conditions) can be used in the case of weakly mixing sets. It was proved in [Banks 1999] that most of the conditions that can be expressed in terms of intersections of sets $N(U, V)$ lead to weak mixing. Of particular interest is the condition, proved first in [Petersen 1970], which says that a dynamical system is weakly mixing if and only if $N(U, V) \cap N(U, U) \neq \varnothing$ for any nonempty open sets $U, V \subset X$. In the spirit of the above fact, we find the following criterion for weak mixing of order $n$. It will be shown later, in Example 3.2, that we cannot use exactly the same condition as in [Petersen 1970].

Theorem 3.1. Let $(X, f)$ be a dynamical system and $A \subset X$ a nontrivial closed subset with $n \geq 2$. Then $A$ is a weakly mixing set of order $n$ if and only if for any $n+1$ open subsets $U_{1}, V_{1}, V_{2}, \ldots, V_{n}$ of $X$ intersecting $A$,

$$
N\left(U_{1} \cap A, V_{1}\right) \cap \bigcap_{i=2}^{n} N\left(V_{i} \cap A, V_{i}\right) \neq \varnothing .
$$

It is shown in [Li 2011, Theorem 3.2] that if a dynamical system $(X, f)$ is weakly mixing, then there exists a residual subset $K$ of $X$ such that for every $x \in K$ and every nonempty open subset $U$ of $X$, the set $N(x, U)$ contains an IP-set. 
This theorem was generalized in [Oprocha and Zhang 2013, Theorem 8], which states that if $A$ is a weakly mixing set of order 2 and $U$ is an open subset of $X$ intersecting $A$, then there is an $x \in U \cap A$ such that for every open subset $V$ of $X$ intersecting $A$ the set $N(x, V)$ contains an IP-set. Using the idea in the proof of [Li 2011, Theorem 3.2], we could extend the above fact from [Oprocha and Zhang 2013] a little further.

Theorem 3.4. Let $(X, f)$ be a dynamical system and $A \subset X$ a weakly mixing set of order $n$ with $n \geq 2$. Then there exists a residual subset $K$ of $A$ such that for any $x \in K$ and any choice of $n-1$ open subsets $U_{1}, \ldots, U_{n-1}$ of $X$ intersecting $A$ there exist points $y_{i} \in U_{i} \cap A$, where $i=1, \ldots, n-1$, such that $N\left(x, U_{1}\right) \cap \bigcap_{i=1}^{n-1} N\left(y_{i}, U_{i}\right)$ contains an IP-set.

A subset $A$ of $X$ is transitive in $(X, f)$ if, for any open subsets $U$ and $V$ of $X$ intersecting $A$, the set $N(U \cap A, V)$ is not empty; $A$ is totally transitive if it is transitive in $\left(X, f^{k}\right)$ for every $k \in \mathbb{N}$. Let $n \geq 2$ be an integer. It is clear that a nontrivial closed subset $A \subset X$ is weakly mixing of order $n$ if and only if $A^{n}$ is a transitive set in $\left(X^{n}, f^{(n)}\right)$. Using Theorem 3.4, we have the following result.

Proposition 3.6. Let $(X, f)$ be a dynamical system and $A \subset X$ a nontrivial closed subset.

(1.3.1) If $A$ is a weakly mixing set of order 2 , then $A$ is totally transitive.

(1.3.2) If $A$ is a weakly mixing set of order $n$ for $f$ with $n \geq 3$, then, for every $k \in \mathbb{N}$, $A$ is a weakly mixing set of order $n-1$ for $f^{k}$.

The authors in [Huang et al. 2012] proved that a dynamical system is weakly mixing if and only if it has the IP-independent property (a formal definition of independence will be given later). We will obtain a similar result for the case of weakly mixing sets.

Inspired by the result of Xiong and Yang [1991], Blanchard and Huang [2008] provided an alternative definition of a weakly mixing set. Strictly speaking, it was proved in [Blanchard and Huang 2008] that a nontrivial closed set $A \subset X$ is a weakly mixing set if and only if there exists a dense Mycielski subset $B$ of $A$ such that for any $C \subset B$ and any continuous map $g: C \rightarrow A$ there exists an increasing sequence of natural numbers $\left\{n_{i}\right\}_{i=1}^{\infty}$ for which $\lim _{i \rightarrow \infty} f^{n_{i}}(x)=g(x)$ for any $x \in C$.

Similarly, we can introduce Xiong chaotic sets of a finite order as follows. A subset $K$ of $X$ with at least $n$ points is called a Xiong chaotic set of order $n$ if for any subset $E$ of $K$ with cardinality $n$ and for any map $g: E \rightarrow \bar{K}$ there is an increasing subsequence $\left\{q_{i}\right\}_{i=1}^{\infty}$ in $\mathbb{N}$ such that $\lim _{i \rightarrow \infty} f^{q_{i}}(x)=g(x)$ for every $x \in E$. Later we will show that a result analogous to [Blanchard and Huang 2008] holds; that is, any nontrivial closed subset $A$ of $X$ is a weakly mixing set of order $n$ if and only if there exists a dense Mycielski subset $S$ of $A$ which is Xiong chaotic 
of order $n$. A dynamical system has a weakly mixing set of order $n$ if and only if it has an uncountable Xiong chaotic set of order $n$. An advantage of Xiong chaotic sets is that they are hereditary by subsets, while weakly mixing sets are not.

For a dynamical system $(X, f)$, the proximal relation is

$$
\operatorname{Prox}_{2}(f)=\left\{(x, y) \in X \times X: \liminf _{k \rightarrow \infty} d\left(f^{k}(x), f^{k}(y)\right)=0\right\},
$$

and the proximal cell of a point $x \in X$ is $\operatorname{Prox}_{2}(f)(x)=\left\{y \in X:(x, y) \in \operatorname{Prox}_{2}(f)\right\}$. It was shown in [Akin and Kolyada 2003] that if $(X, f)$ is weakly mixing, then, for every $x \in X$, the proximal cell $\operatorname{Prox}_{2}(f)(x)$ of $x$ is residual in $X$. The authors in [Huang et al. 2004] studied the structure of proximal cells of points in weakly mixing systems and showed that there is a Xiong chaotic set in those proximal cells. In [Oprocha and Zhang 2013] it was proved that for every closed weakly mixing set $A$ and every $x \in A$, the set $\operatorname{Prox}_{2}(f)(x) \cap A$ is residual in $A$. We will show that the same is true if we consider proximal tuples instead of pairs. For a dynamical system $(X, f)$ and a positive integer $n \geq 2$, the $n$-th proximal relation is

$$
\operatorname{Prox}_{n}(f)=\left\{\left(x_{1}, \ldots, x_{n}\right) \in X^{n}: \liminf _{k \rightarrow \infty} \max _{1 \leq i<j \leq n} d\left(f^{k}\left(x_{i}\right), f^{k}\left(x_{j}\right)\right)=0\right\},
$$

and the $n$-th proximal cell of a point $x_{0} \in X$ is

$$
\operatorname{Prox}_{n}(f)\left(x_{0}\right)=\left\{\left(x_{1}, \ldots, x_{n-1}\right) \in X^{n-1}:\left(x_{0}, x_{1}, \ldots, x_{n-1}\right) \in \operatorname{Prox}_{n}(f)\right\} .
$$

Theorem 5.6. Let $(X, f)$ be a dynamical system and $A \subset X$ a weakly mixing set. Then for every $x_{0} \in A$ and $n \geq 2$, the set $\operatorname{Prox}_{n}(f)\left(x_{0}\right) \cap A^{n-1}$ is residual in $A^{n-1}$.

In fact, we prove even more in the following theorem, where $L Y_{n}^{\delta}(X, f)\left(x_{0}\right)$ is the $n$-scrambled cell of $x_{0}$ with modular $\delta>0$. More precisely, $L Y_{n}^{\delta}(X, f)\left(x_{0}\right)$ is the collection of points $\left(x_{1}, \ldots, x_{n-1}\right)$ in $X^{n-1}$ such that $\left(x_{0}, x_{1}, \ldots, x_{n-1}\right)$ is proximal and

$$
\limsup _{k \rightarrow \infty} \min _{0 \leq i<j \leq n-1} d\left(f^{k}\left(x_{i}\right), f^{k}\left(x_{j}\right)\right) \geq \delta
$$

Theorem 5.7. Let $(X, f)$ be a dynamical system and $A \subset X$ a weakly mixing set. Then, for every $n \geq 2$, there exists a $\delta>0$ such that, for every $x_{0} \in A$, it holds that $L Y_{n}^{\delta}(X, f)\left(x_{0}\right) \cap A^{n-1}$ is residual in $A^{n-1}$.

The following result shows that, when we look only at separation of trajectories of tuples, weak mixing of order 2 is enough to obtain rich structure of such points (see Section 2B for definitions of sensitivity).

Theorem 3.7. Let $(X, f)$ be a dynamical system and $A \subset X$ a weakly mixing set of order 2. Then $A$ is a sensitive set in $(\overline{\operatorname{Orb}(A, f)}, f)$. In particular, the system $(\overline{\operatorname{Orb}(A, f)}, f)$ is $n$-sensitive for every $n \geq 2$. 
In the final section, we prove that on topological graphs weakly mixing sets of order 2 can be approximated arbitrarily closely (in the Hausdorff metric) by a weakly mixing set of all orders. This completes our previous research in [Oprocha and Zhang 2011].

\section{Preliminaries}

In this section, we provide some basic notation, definitions and results which will be used later in this paper. Denote by $\mathbb{N}, \mathbb{N}_{0}, \mathbb{Z}$ and $\mathbb{R}$ the set of all positive integers, nonnegative integers, integers and real numbers, respectively. A subset $A$ of $\mathbb{N}$ is an $I P$-set if there exists a sequence $\left\{p_{j}\right\}_{j=1}^{\infty}$ in $\mathbb{N}$ such that $A=F S\left(\left\{p_{j}\right\}_{j=1}^{\infty}\right)$, where

$$
F S\left(\left\{p_{j}\right\}_{j=1}^{\infty}\right)=\left\{\sum_{j \in \alpha} p_{j}: \alpha \text { is a nonempty finite subset of } \mathbb{N}\right\}
$$

is the set of finite sums of $\left\{p_{j}\right\}_{j=1}^{\infty}$.

Let $X$ be a compact metric space. A subset $C$ of $X$ is a Cantor set if it is homeomorphic to the standard Cantor ternary set (equivalently, it is a perfect and totally disconnected compact metric space). We say that a subset $K$ of $X$ is a Mycielski set if it can be presented as a countable union of Cantor sets. The next two facts help to deal with residual relations. They are important tools with numerous applications. See [Akin 2004] for a comprehensive treatment of this topic.

Lemma 2.1 (Ulam lemma). Let $X$ be a perfect compact metric space. If $R$ is a dense $G_{\delta}$ subset of $X^{n}$, then there exists a dense $G_{\delta}$ subset $K$ of $X$ such that, for every $x \in K$, the set $R(x)=\left\{\left(x_{1}, \ldots, x_{n-1}\right) \in X^{n-1}:\left(x, x_{1}, \ldots, x_{n-1}\right) \in R\right\}$ is residual in $X^{n-1}$.

Theorem 2.2 (Mycielski theorem [1964]). Let X be a perfect compact metric space. If $R$ is a dense $G_{\delta}$ subset of $X^{n}$, then there exists a dense Mycielski subset $K$ of $X$ such that, for any $n$ distinct points $x_{1}, \ldots, x_{n} \in K$, we have $\left(x_{1}, x_{2}, \ldots, x_{n}\right) \in R$.

2A. Topological dynamics. By a (topological) dynamical system we mean a pair $(X, f)$ consisting of a compact metric space $(X, d)$ and a continuous map $f: X \rightarrow X$. If $X$ is a singleton, then we say that $(X, f)$ is trivial. If $K \subset X$ is a nonempty closed subset satisfying $f(K) \subset K$, then we say that $(K, f)$ is a subsystem of $(X, f)$.

Let $(X, f)$ be a dynamical system with $\varnothing \neq A \subset X$ and $x \in X$. The set

$$
\operatorname{Orb}(A, f)=\bigcup_{n \in \mathbb{N}_{0}} f^{n}(A)
$$

is said to be the (positive) orbit of $A$ under $f$. Clearly, $(\overline{\operatorname{Orb}(A, f)}, f)$ is a subsystem of $(X, f)$. We will write $\operatorname{Orb}(x, f)=\operatorname{Orb}(\{x\}, f)$ for short. 
We say that a point $x \in X$ is a periodic point of $(X, f)$ if $f^{n}(x)=x$ for some $n \in \mathbb{N}$, a recurrent point of $(X, f)$ if there exists an increasing sequence $\left\{k_{n}\right\}_{n=1}^{\infty}$ in $\mathbb{N}$ such that $\lim _{n \rightarrow \infty} f^{k_{n}}(x)=x$, and a transitive point of $(X, f)$ if $\operatorname{Orb}(x, f)$ is dense in $X$. Denote by $\operatorname{Per}(X, f), \operatorname{Rec}(X, f)$ and $\operatorname{Tran}(X, f)$ the set of all periodic points, recurrent points and transitive points, respectively, of $(X, f)$. A dynamical system $(X, f)$ is minimal if $\operatorname{Tran}(X, f)=X$. A point $x \in X$ is minimal if the subsystem $(\overline{\operatorname{Orb}(x, f)}), f)$ is minimal.

Let $(X, f)$ be a dynamical system and $A \subset X$ with $n \geq 2$. Define the sets $A^{n}=\left\{\left(x_{1}, x_{2}, \ldots, x_{n}\right): x_{1}, \ldots, x_{n} \in A\right\}$ and $\Delta_{n}(A)=\left\{(x, x, \ldots, x) \in A^{n}: x \in A\right\}$. The map $f^{(n)}$ is induced on $X^{n}$ by the formula

$$
f^{(n)}\left(x_{1}, x_{2}, \ldots, x_{n}\right)=\left(f\left(x_{1}\right), f\left(x_{2}\right), \ldots, f\left(x_{n}\right)\right) .
$$

Let $(X, f)$ be a dynamical system with $x \in X$ and $A, B \subset X$. Define the sets $N(x, A)=\left\{n \in \mathbb{N}: f^{n}(x) \in A\right\}$ and $N(A, B)=\left\{n \in \mathbb{N}: f^{n}(A) \cap B \neq \varnothing\right\}$. When we want to emphasize the map $f$, we instead use $N_{f}(x, A)$ and $N_{f}(A, B)$. A dynamical system $(X, f)$ is called transitive if, for any nonempty open subsets $U$ and $V$ of $X$, the set $N(U, V)$ is not empty, totally transitive if $\left(X, f^{k}\right)$ is transitive for every $k \in \mathbb{N}$, and weakly mixing if $\left(X^{2}, f^{(2)}\right)$ is transitive. It is well known that if $(X, f)$ is transitive, then $\operatorname{Tran}(X, f)$ is a dense $G_{\delta}$ subset of $X$.

2B. Proximal and scrambled tuples. We say that an $n$-tuple $\left(x_{1}, \ldots, x_{n}\right) \in X^{n}$ (where $n \geq 2$ ) is proximal if

$$
\liminf _{k \rightarrow \infty} \max _{1 \leq i<j \leq n} d\left(f^{k}\left(x_{i}\right), f^{k}\left(x_{j}\right)\right)=0 .
$$

Let $\operatorname{Prox}_{n}(f)$ denote the collection of all proximal $n$-tuples in $(X, f)$. It is easy to verify that $\operatorname{Prox}_{n}(f)$ is a $G_{\delta}$ subset of $X^{n}$. For $x \in X$, define the $n$-th proximal cell of $x$ as

$$
\operatorname{Prox}_{n}(f)(x)=\left\{\left(x_{1}, \ldots, x_{n-1}\right) \in X^{n-1}:\left(x, x_{1}, \ldots, x_{n-1}\right) \text { is proximal }\right\} .
$$

An $n$-tuple $\left(x_{1}, \ldots, x_{n}\right) \in X^{n}$ (where $n \geq 2$ ) is called scrambled (with modular $\delta>0)$ if it is proximal and

$$
\limsup _{k \rightarrow \infty} \min _{1 \leq i<j \leq n} d\left(f^{k}\left(x_{i}\right), f^{k}\left(x_{j}\right)\right) \geq \delta .
$$

A subset $S$ of $X$ is called $n$-scrambled if any $n$ distinct points in $S$ form a scrambled $n$-tuple. The system $(X, f)$ is called Li-Yorke $n$-chaotic if there exists an uncountable $n$-scrambled subset $S$ of $X$.

Xiong [2005] introduced the concept of $n$-sensitivity. Specifically, a dynamical system $(X, f)$ is called $n$-sensitive, where $n \geq 2$, if there exists a $\delta>0$ such that for every nonempty open set $U \subset X$ there are distinct points $x_{1}, x_{2}, \ldots, x_{n} \in U$ 
and some $m \in \mathbb{N}$ with

$$
\min _{1 \leq i<j \leq n} d\left(f^{m}\left(x_{i}\right), f^{m}\left(x_{j}\right)\right)>\delta .
$$

This definition was further generalized in [Ye and Zhang 2008] to sensitive sets. A subset $A$ of $X$ is sensitive if for any $n \geq 2$, any $n$ distinct points $x_{1}, x_{2}, \ldots, x_{n}$ in $A$, any neighborhood $U_{i}$ of $x_{i}, i=1,2, \ldots, n$, and any nonempty open set $U \subset X$ there exists a $k \in \mathbb{N}$ and $y_{i} \in U$ such that $f^{k}\left(y_{i}\right) \in U_{i}$ for $i=1,2, \ldots, n$. It is shown in [Ye and Zhang 2008] that a transitive system is $n$-sensitive if and only if there exists a sensitive set with cardinality $n$. Note that 2 -scrambled set, Li-Yorke 2-chaos and 2-sensitivity are classical definitions.

2C. Transitive sets and weakly mixing sets. Let $(X, f)$ be a dynamical system. A subset $A$ of $X$ is transitive in $(X, f)$ if for any open subsets $U$ and $V$ of $X$ intersecting $A$, the set $N(U \cap A, V)$ is not empty and totally transitive if $A$ is transitive in $\left(X, f^{k}\right)$ for every $k \in \mathbb{N}$. Let $n \geq 2$ be an integer. A nontrivial closed subset $A \subset X$ is weakly mixing of order $n$ provided that $A^{n}$ is a transitive set in $\left(X^{n}, f^{(n)}\right)$ and weakly mixing of all orders or simply weakly mixing if $A$ is weakly mixing of order $k$ for all $k=2,3, \ldots$.

Remark 2.3. In the present paper we require a weakly mixing set (of order $n$ ) to be closed and nontrivial which is a little more restrictive than the original definition in [Oprocha and Zhang 2011].

The following result is derived directly from the definition.

Lemma 2.4. Let $(X, f)$ be a dynamical system and $A \subset X$ a nontrivial closed subset with $n \geq 2$. Then $A$ is weakly mixing of order $n$ if and only if, for any open subsets $U_{1}, \ldots, U_{n}$ and $V_{1}, \ldots, V_{n}$ of $X$ intersecting $A$,

$$
\bigcap_{i=1}^{n} N\left(U_{i} \cap A, V_{i}\right) \neq \varnothing .
$$

The following lemmas, while simple in proof, are very useful in practice. The proofs can be found in [Oprocha and Zhang 2011; 2014].

Lemma 2.5. Let $(X, f)$ be a dynamical system and $A \subset X$ a weakly mixing subset of order 2. Then A is perfect.

Lemma 2.6. Let $(X, f)$ be a dynamical system and $A$ a closed subset of $X$. If $A$ is a transitive set, then:

(2.6.1) $(\overline{\operatorname{Orb}(A, f)}, f)$ is a transitive subsystem of $(X, f)$.

(2.6.2) $A \cap \operatorname{Tran}(\overline{\operatorname{Orb}(A, f)}, f)$ is a dense $G_{\delta}$ subset of $A$. 
2D. Symbolic dynamics. Let $\mathcal{A}$ be a finite set (an alphabet) endowed with the discrete topology and let $\mathcal{A}^{\mathbb{N}_{0}}$ denote the Cantor space with respect to the product topology. We write elements of $\mathcal{A}^{\mathbb{N}_{0}}$ as $x=x_{0} x_{1} \cdots$. The shift transformation $\sigma: \mathcal{A}^{\mathbb{N}_{0}} \rightarrow \mathcal{A}^{\mathbb{N}_{0}}$ is given by $\sigma(x)_{i}=x_{i+1}$ for $i \in \mathbb{N}_{0}$. The dynamical system $\left(\mathcal{A}^{\mathbb{N}_{0}}, \sigma\right)$ is called the full shift over $\mathcal{A}$.

By a word (over $\mathcal{A}$ ), we mean any finite sequence $u=u_{0} \cdots u_{n-1}, n \geq 1$ where $u_{i} \in \mathcal{A}$. The length of $u$ is denoted by $|u|=n$ and the set of all words is denoted by $\mathcal{A}^{+}$. If $x \in \mathcal{A}^{\mathbb{N}_{0}}$ and $0 \leq i<j$, then by $x_{[i, j]}$ we mean the sequence $x_{i} x_{i+1} \cdots x_{j}$. For simplicity, we use the notation $x_{[i, j)}=x_{[i, j-1]}$. If $a_{1} \cdots a_{m} \in \mathcal{A}^{+}$, then we define the cylinder set

$$
C\left[a_{1} \cdots a_{m}\right]=\left\{x \in \mathcal{A}^{\mathbb{N}_{0}}: x_{[0, m)}=a_{1} \cdots a_{m}\right\} .
$$

If $X$ is a subshift, we denote the cylinder set by $C_{X}\left[a_{1} \cdots a_{m}\right]=C\left[a_{1} \cdots a_{m}\right] \cap X$.

2E. Topological graphs. Roughly speaking, a topological graph is a continuum which is the union of a finite number of intervals which can intersect only at endpoints and do not have self-intersections. More formally, a topological graph is a compact connected metric space $G$ which is homeomorphic to a polyhedron (a geometric realization) of some finite one-dimensional complex. In particular, we can naturally endow $G$ with the metric $d$ given by the length of the shortest arc joining $x, y$ in $G$ (induced on $G$ from the polyhedron). An arc $I \subset G$ is a closed interval if there is a homeomorphism $\varphi:[0,1] \rightarrow I$ such that the set $\varphi((0,1))$ is open in $G$.

Let $(G, f)$ be a dynamical system and let $I, J \subset G$ be closed intervals. If there exists a closed interval $K \subset I$ such that $f(K)=J$, then we say that $I f$-covers $J$ and denote this fact by $I \stackrel{f}{\Longrightarrow} J$. We will need the following standard properties of $f$-covering (see [Alsedà et al. 2003, p. 590]):

Lemma 2.7. Let $I, J, K, L \subset G$ be closed intervals and let $f, g: G \rightarrow G$ be continuous.

(2.7.1) If $I \subset K, L \subset J$ and $I \stackrel{f}{\Longrightarrow} J$, then $K \stackrel{f}{\Longrightarrow} L$.

(2.7.2) If $I \stackrel{f}{\Longrightarrow} J$ and $J \stackrel{g}{\Longrightarrow} K$, then $I \stackrel{g \circ f}{\Longrightarrow} K$.

(2.7.3) If $J \subset f(I)$, and $K_{1}, K_{2} \subset J$ are closed intervals such that $K_{1} \cap K_{2}$ is at most one point, then $I \stackrel{f}{\Longrightarrow} K_{1}$ or $I \stackrel{f}{\Longrightarrow} K_{2}$.

\section{Weakly mixing sets of finite order}

In this section we study weakly mixing sets of finite order. It is clear that a dynamical system $(X, f)$ is weakly mixing if and only if, for any four nonempty open subsets $U_{1}, V_{1}, U_{2}, V_{2}$ of $X$, we have $N\left(U_{1}, V_{1}\right) \cap N\left(U_{2}, V_{2}\right) \neq \varnothing$. It is shown in [Petersen 1970] that we can reduce four open sets in the characterization of weak mixing to two open sets; that is, a dynamical system $(X, f)$ is weakly mixing 
if and only if, for any two nonempty open subsets $U$ and $V$ of $X$, it holds that $N(U, V) \cap N(U, U) \neq \varnothing$ (this was later extended in [Banks 1999] to show that most of the possible conditions of this kind are equivalent to weak mixing). Similar to the above condition, we can simplify the condition in Lemma 2.4 to obtain an alternative definition of weakly mixing set of order $n$. The advantage is that we have to verify conditions on transfer times for only $n+1$ open sets instead of $2 n$ sets in the original definition.

Theorem 3.1. Let $(X, f)$ be a dynamical system and $A \subset X$ a nontrivial closed subset with $n \geq 2$. Then $A$ is a weakly mixing set of order $n$ if and only if, for any $n+1$ open subsets $U_{1}, V_{1}, V_{2}, \ldots, V_{n}$ of $X$ intersecting $A$,

$$
N\left(U_{1} \cap A, V_{1}\right) \cap \bigcap_{i=2}^{n} N\left(V_{i} \cap A, V_{i}\right) \neq \varnothing .
$$

Proof. The necessity follows from Lemma 2.4. Now we prove the sufficiency. Fix any $2 n$ open subsets $U_{1}, V_{1}, U_{2}, V_{2}, \ldots, U_{n}, V_{n}$ of $X$ intersecting $A$. Assume that for some $1 \leq j<n$ we have

$$
\bigcap_{i=1}^{j} N\left(U_{i} \cap A, V_{i}\right) \cap \bigcap_{l=j+1}^{n} N\left(V_{l} \cap A, V_{l}\right) \neq \varnothing .
$$

Then there is a $k>0$ and open subsets $U_{1}^{\prime}, \ldots, U_{j}^{\prime}, V_{j+1}^{\prime}, \ldots V_{n}^{\prime}$ of $X$ intersecting $A$ such that $U_{i}^{\prime} \subset U_{i}$ and $f^{k}\left(U_{i}^{\prime}\right) \subset V_{i}$ for each $i=1, \ldots, j$, and $V_{l}^{\prime} \subset V_{l}$ and $f^{k}\left(V_{l}^{\prime}\right) \subset V_{l}$ for each $l=j+1, \ldots, n$. By the assumption we can choose

$$
m \in N\left(U_{j+1} \cap A, V_{j+1}^{\prime}\right) \cap \bigcap_{i=1}^{j} N\left(U_{i}^{\prime} \cap A, U_{i}^{\prime}\right) \cap \bigcap_{l=j+2}^{n} N\left(V_{l}^{\prime} \cap A, V_{l}^{\prime}\right),
$$

so that

$$
m+k \in \bigcap_{i=1}^{j+1} N\left(U_{i} \cap A, V_{i}\right) \cap \bigcap_{l=j+2}^{n} N\left(V_{l} \cap A, V_{l}\right) .
$$

Hence, by induction on $j$, we eventually obtain that

$$
\bigcap_{i=1}^{n} N\left(U_{i} \cap A, V_{i}\right) \neq \varnothing,
$$

which implies that $A$ is weakly mixing of order $n$.

Unfortunately, the above technique is not sufficient if we want to directly copy the condition from [Petersen 1970]. This condition simply will not induce even the smallest degree of local weak mixing, as shown by the next example. The technique 
used here is a modification of Example 6.1 from [Oprocha and Zhang 2011]. Since the construction is somewhat long and complicated, we move it to the Appendix.

Example 3.2. There are a dynamical system $(X, f)$ and a nontrivial closed subset $A$ of $X$ satisfying the following two conditions:

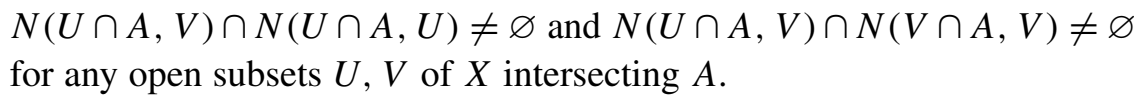
for any open subsets $U, V$ of $X$ intersecting $A$.

(3.2.2) $A$ is not weakly mixing of order 2 .

It is shown in [Li 2011, Theorem 3.2] that if a dynamical system $(X, f)$ is weakly mixing, then there exists a residual subset $K$ of $X$ such that, for every $x \in K$ and every nonempty open subset $U$ of $X$, the set $N(x, U)$ contains an IP-set. This theorem was generalized in [Oprocha and Zhang 2013, Theorem 8], which states that if $A$ is a weakly mixing set of order 2 and $U$ is an open set of $X$ intersecting $A$, then there is an $x \in U \cap A$ such that for every open set $V$ of $X$ intersecting $A$ the set $N(x, V)$ contains an IP-set. The following lemma is inspired by the proof of [Li 2011, Theorem 3.2]. It allows us to extend the above fact from [Oprocha and Zhang 2013] a little further.

Lemma 3.3. Let $(X, f)$ be a dynamical system with $n \geq 2$. If there are $n$ points $x, y_{1}, y_{2}, \ldots, y_{n-1} \in X$ with $x \neq y_{1}$ such that

$$
\left.\left(y_{1}, y_{1}, y_{2}, \ldots, y_{n-1}\right) \in \overline{\operatorname{Orb}\left(\left(x, y_{1}, \ldots, y_{n-1}\right), f^{(n)}\right.}\right),
$$

then, for every choice of open neighborhoods $U_{i}$ of $y_{i}, i=1,2, \ldots, n-1$, the set

$$
N\left(x, U_{1}\right) \cap \bigcap_{i=1}^{n-1} N\left(y_{i}, U_{i}\right)
$$

contains an IP-set.

Proof. For each $i=1,2, \ldots, n-1$ fix an open neighborhood $U_{i}$ of $y_{i}$. Since $x \neq y_{1}$, we may assume that $x \notin U_{1}$. We are going to construct an IP-set in $N\left(x, U_{1}\right) \cap \bigcap_{i=1}^{n-1} N\left(y_{i}, U_{i}\right)$. We start our construction by setting $U_{i}^{(1)}=U_{i}$ for $i=1, \ldots, n-1$.

By (1), there exists a $p_{1} \in \mathbb{N}$ such that $f^{p_{1}}(x) \in U_{1}^{(1)}$ and $f^{p_{1}}\left(y_{i}\right) \in U_{i}^{(1)}$ for $i=1, \ldots, n-1$. Let $U_{i}^{(2)}=U_{i}^{(1)} \cap f^{-p_{1}}\left(U_{i}^{(1)}\right)$ for $i=1, \ldots, n-1$. Clearly, $U_{i}^{(2)}$ is also an open neighborhood of $y_{i}$ for $i=1, \ldots, n-1$. By (1) again there exists a $p_{2}>0$ such that $f^{p_{2}}(x) \in U_{1}^{(2)}$ and $f^{p_{2}}\left(y_{i}\right) \in U_{i}^{(2)}$ for $i=1, \ldots, n-1$. Then, for every $m \in F S\left(\left\{p_{j}\right\}_{j=1}^{2}\right)$, we have $f^{m}(x) \in U_{1}$ and $f^{m}\left(y_{i}\right) \in U_{i}$ for $i=1, \ldots, n-1$. We continue this construction inductively.

Assume that for some $k \geq 2$ positive integers $p_{1}, p_{2}, \ldots, p_{k}$ have been constructed in such a way that if $m \in F S\left(\left\{p_{j}\right\}_{j=1}^{k}\right)$ then $f^{m}(x) \in U_{1}$ and $f^{m}\left(y_{i}\right) \in U_{i}$ 
for $i=1, \ldots, n-1$. For each $i=1, \ldots, n-1$ set

$$
U_{i}^{(k+1)}=U_{i} \cap \bigcap_{m \in F S\left(\left\{p_{j}\right\}_{j=1}^{k}\right)} f^{-m}\left(U_{i}\right)
$$

and observe that each $U_{i}^{(k+1)}$ is also an open neighborhood of $y_{i}$ for $i=1, \ldots, n-1$. By (1) there exists a $p_{k+1}>0$ such that $f^{p_{k+1}}(x) \in U_{1}^{(k+1)}$ and $f^{p_{k+1}}\left(y_{i}\right) \in U_{i}^{(k+1)}$ for $i=1, \ldots, n-1$. Then, completing the induction, for every $m \in F S\left(\left\{p_{j}\right\}_{j=1}^{k+1}\right)$, we have $f^{m}(x) \in U_{1}$ and $f^{m}\left(y_{i}\right) \in U_{i}$ for $i=1, \ldots, n-1$. Thus, we get a sequence $\left\{p_{j}\right\}_{j=1}^{\infty}$ such that $F S\left(\left\{p_{j}\right\}_{j=1}^{\infty}\right) \subset N\left(x, U_{1}\right) \cap \bigcap_{i=1}^{n-1} N\left(y_{i}, U_{i}\right)$.

Theorem 3.4. Let $(X, f)$ be a dynamical system and $A \subset X$ a weakly mixing set of order $n$ with $n \geq 2$. Then there exists a residual subset $K$ of $A$ such that for any $x \in K$ and any choice of $n-1$ open subsets $U_{1}, \ldots, U_{n-1}$ of $X$ intersecting $A$ there exist points $y_{i} \in U_{i} \cap A$, where $i=1, \ldots, n-1$, such that $N\left(x, U_{1}\right) \cap \bigcap_{i=1}^{n-1} N\left(y_{i}, U_{i}\right)$ contains an IP-set.

Proof. Since $A^{n}$ is a transitive set in $\left(X^{n}, f^{(n)}\right)$, we have by Lemma 2.6 that the relation $\left.R=A^{n} \cap \operatorname{Tran}\left(\overline{\operatorname{Orb}\left(A^{n}, f^{(n)}\right.}\right), f^{(n)}\right)$ is a dense $G_{\delta}$ subset of $A^{n}$. By the Ulam lemma, there exists a dense $G_{\delta}$ subset $K$ of $A$ such that for every $x \in K$ the section of $R$ at $x$, that is, the set $R(x)=\left\{\left(y_{1}, \ldots, y_{n-1}\right) \in A^{n-1}:\left(x, y_{1}, \ldots, y_{n-1}\right) \in R\right\}$, is residual in $A^{n-1}$. It remains to show that $K$ satisfies our requirement.

Fix $x \in K$ and $n-1$ open subsets $U_{1}, U_{2}, \ldots, U_{n-1}$ of $X$ intersecting $A$. Since $R(x)$ is residual, we can select points $y_{i} \in U_{i} \cap A$, where $i=1, \ldots, n-1$, such that $\left(x, y_{1}, \ldots, y_{n-1}\right) \in R$ and $x \neq y_{1}$ (recall that $A$ is perfect by Lemma 2.5). By the definition of $R$ we obtain

$$
\left.\left(y_{1}, y_{1}, y_{2}, \ldots, y_{n-1}\right) \in \overline{\operatorname{Orb}\left(\left(x, y_{1}, \ldots, y_{n-1}\right), f^{(n)}\right.}\right) .
$$

Now the result follows by Lemma 3.3.

Corollary 3.5. Let $(X, f)$ be a dynamical system and $A \subset X$ a weakly mixing set of order $n$ with $n \geq 2$. Then, for any $n$ open subsets $U_{1}, V_{1}, V_{2}, \ldots, V_{n-1}$ of $X$ intersecting A,

$$
N\left(U_{1} \cap A, V_{1}\right) \cap \bigcap_{i=1}^{n-1} N\left(V_{i} \cap A, V_{i}\right)
$$

contains an IP-set.

It is shown in [Oprocha and Zhang 2012, Theorem 6] that a weakly mixing set of order 2 is totally transitive. Now, with the help of Corollary 3.5, we can extend it as follows.

Proposition 3.6. Let $(X, f)$ be a dynamical system and $A \subset X$ a nontrivial closed subset. 
(3.6.1) If $A$ is a weakly mixing set of order 2 , then $A$ is totally transitive.

(3.6.2) If $A$ is a weakly mixing set of order $n$ for $f$ with $n \geq 3$, then, for every $k \in \mathbb{N}$, $A$ is a weakly mixing set of order $n-1$ for $f^{k}$.

Proof. If $F$ contains an IP-set, then $F \cap n \mathbb{N} \neq \varnothing$ for every $n \in \mathbb{N}$. Now the result follows by Theorem 3.1 and Corollary 3.5.

The above fact motivates us to state the following question for investigation.

Question. Let $(X, f)$ be a dynamical system and $k \in \mathbb{N}, n \geq 2$. If a subset $A \subset X$ is weakly mixing of order $n$ for $f$, is it weakly mixing of order $n$ for $f^{k}$ ?

Theorem 3.7. Let $(X, f)$ be a dynamical system and $A \subset X$ a weakly mixing set of order 2. Then $A$ is a sensitive set in $(\overline{\operatorname{Orb}(A, f)}, f)$. In particular, the system $(\overline{\operatorname{Orb}(A, f)}, f)$ is $n$-sensitive for every $n \geq 2$.

Proof. Without loss of generality, we assume that $\overline{\operatorname{Orb}(A, f)}=X$. First note that both $A$ and $X$ must be perfect. Now let $n \geq 2$ and fix $n$ distinct points $x_{1}, x_{2}, \ldots, x_{n}$ in $A$. Let $U$ be a nonempty open subset of $X$ and $U_{i}$ an open neighborhood of $x_{i}$ for $i=1,2, \ldots, n$. There is some $k \geq 0$ such that $f^{k}(A) \cap U \neq \varnothing$ and therefore there is an open subset $V$ of $X$ intersecting $A$ such that $f^{k}(V) \subset U$. Since $A$ is a weakly mixing set of order 2 , there exists an $m_{2} \in \mathbb{N}$ such that $U_{1} \cap A \cap f^{-m_{2}}\left(U_{2}\right) \neq \varnothing$ and $V \cap A \cap f^{-m_{2}}(V) \neq \varnothing$. By induction, there exist $m_{3}, \ldots, m_{n} \in \mathbb{N}$ such that

$$
U_{1} \cap A \cap \bigcap_{i=2}^{n} f^{-m_{i}}\left(U_{i}\right) \neq \varnothing \quad \text { and } \quad V \cap A \cap \bigcap_{i=2}^{n} f^{-m_{i}}(V) \neq \varnothing .
$$

And so there is a point $y \in A$ such that $\left\{y, f^{m_{2}}(y), \ldots, f^{m_{n}}(y)\right\} \subset V$. By Lemma 2.6, $\operatorname{Tran}(X, f) \cap A$ is a dense $G_{\delta}$ subset of $A$, and therefore we can choose $x$ in $\operatorname{Tran}(X, f) \cap U_{1} \cap A \cap \bigcap_{i=2}^{n} f^{-m_{i}}\left(U_{i}\right)$; that is, we choose an $x \in U_{1}$ such that $f^{m_{i}}(x) \in U_{i}$ for $i=2, \ldots, n$. Since $x$ is a transitive point in $(X, f)$ and the space $X$ is perfect, there exists a $p \in \mathbb{N}_{0}$ such that $\left\{f^{p}(x), f^{p+m_{2}}(x), \ldots, f^{p+m_{n}}(x)\right\} \subset V$ and a $q>p+k$ such that $f^{q}(x) \in U_{1}$ and $f^{q+m_{i}}(x) \in U_{i}$ for $i=2, \ldots, n$. Define $r=q-p-k, y_{1}=f^{p+k}(x)$ and $y_{i}=f^{p+k+m_{i}}(x)$ for $i=2, \ldots, n$. Then $y_{i} \in U$ and $f^{r}\left(y_{i}\right) \in U_{i}$ for $i=1,2, \ldots, n$, which implies that $A$ is a sensitive set. Finally, we have by Lemma 2.6 that $(\overline{\operatorname{Orb}(A, f)}, f)$ is $n$-sensitive for every $n \geq 2$.

\section{Xiong chaotic set of finite order}

In this section, we study Xiong chaotic sets of finite order and their connection to weakly mixing sets of finite order.

Definition 4.1. Let $(X, f)$ be a dynamical system with $n \geq 2$. A subset $K$ of $X$ with at least $n$ points is called a Xiong chaotic set of order $n$ if, for any subset $E$ of $K$ 
with cardinality $n$ and for any map $g: E \rightarrow \bar{K}$, there is an increasing subsequence $\left\{q_{i}\right\}_{i=1}^{\infty}$ in $\mathbb{N}$ such that $\lim _{i \rightarrow \infty} f^{q_{i}}(x)=g(x)$ for every $x \in E$.

The following result is straightforward by the definition.

Proposition 4.2. If $K$ is a Xiong chaotic set of order $n$, then there exists a $\delta>0$ such that, for every $n$ distinct points $x_{1}, x_{2}, \ldots, x_{n}$ in $K$,

$$
\begin{gathered}
\liminf _{k \rightarrow \infty} \max _{1 \leq i<j \leq n} d\left(f^{k}\left(x_{i}\right), f^{k}\left(x_{j}\right)\right)=0, \\
\limsup _{k \rightarrow \infty} \min _{1 \leq i<j \leq n} d\left(f^{k}\left(x_{i}\right), f^{k}\left(x_{j}\right)\right)>\delta, \\
\liminf _{k \rightarrow \infty} \max _{1 \leq i \leq n} d\left(f^{k}\left(x_{i}\right), x_{i}\right)=0 .
\end{gathered}
$$

In particular, $K$ is $n$-scrambled with modular $\delta$.

Theorem 4.3. Let $(X, f)$ be a dynamical system and A a perfect subset of $X$ with $n \geq 2$. Then the following conditions are equivalent:

(4.3.1) A is a weakly mixing set of order $n$.

(4.3.2) There exists a dense Mycielski subset $S$ of A which is Xiong chaotic of order $n$.

(4.3.3) There exists a dense subset $S$ of A which is Xiong chaotic of order $n$.

Proof. (4.3.1) $\Rightarrow$ (4.3.2) First note that $A$ is perfect. Since $A^{n}$ is a transitive set in $\left(X^{n}, f^{(n)}\right)$, by Lemma 2.6 the relation $\left.R=A^{n} \cap \operatorname{Tran}\left(\overline{\operatorname{Orb}\left(A^{n}, f^{(n)}\right.}\right), f^{(n)}\right)$ is a dense $G_{\delta}$ subset of $A^{n}$. By the Mycielski theorem, there exists a dense Mycielski subset $S$ of $A$ such that, for every $n$ distinct points $x_{1}, x_{2}, \ldots, x_{n} \in S$, we have $\left(x_{1}, x_{2}, \ldots, x_{n}\right) \in R$. Fix a subset $E$ of $S$ with cardinality $n$ and a map $g: E \rightarrow A$. Enumerate $E$ as $\left\{x_{1}, x_{2}, \ldots, x_{n}\right\}$ and let $y_{i}=g\left(x_{i}\right)$ for $i=1,2, \ldots, n$. Since $\left(x_{1}, x_{2}, \ldots, x_{n}\right)$ is a transitive point in $\left.\left(\overline{\operatorname{Orb}\left(A^{n}, f^{(n)}\right.}\right), f^{(n)}\right)$ and $\left(y_{1}, y_{2}, \ldots, y_{n}\right)$ is in $A^{n}$, there is an increasing subsequence $\left\{q_{k}\right\}_{k=1}^{\infty}$ in $\mathbb{N}$ such that we have $\lim _{k \rightarrow \infty} f^{q_{k}}\left(x_{i}\right)=g\left(x_{i}\right)$ for $i=1,2, \ldots, n$; thus $S$ is a Xiong chaotic set of order $n$.

(4.3.2) $\Rightarrow$ (4.3.3) The implication is trivial.

(4.3.3) $\Rightarrow$ (4.3.1) Fix any open subsets $U_{1}, V_{1}, U_{2}, V_{2}, \ldots, U_{n}, V_{n}$ of $X$ intersecting $A$. Choose $n$ distinct points $x_{i} \in U_{i} \cap S$ and $n$ points $y_{i} \in V_{i} \cap A$ for $i=1,2, \ldots, n$. Define a map $g:\left\{x_{1}, x_{2}, \ldots, x_{n}\right\} \rightarrow A$ as $g\left(x_{i}\right)=y_{i}$ for $i=1,2, \ldots, n$. Then there exists a $k \geq 1$ such that $f^{k}\left(x_{i}\right) \in V_{i}$ for $i=1,2, \ldots, n$. In particular the set $\bigcap_{i=1}^{n} N\left(U_{i} \cap A, V_{i}\right)$ is not empty, which completes the proof.

Corollary 4.4. Let $(X, f)$ be a dynamical system with $n \geq 2$. Then $(X, f)$ has a weakly mixing set of order $n$ if and only if it has an uncountable Xiong chaotic set of order $n$. 
Proof. The necessity follows by Theorem 4.3, since a Mycielski set is uncountable. Now we prove the sufficiency. Let $S$ be an uncountable Xiong chaotic set of order $n$. By compactness of $X$, we can divide the closure $\bar{S}$ of $S$ into $K_{1} \cup K_{2}$, where $K_{1}$ is perfect and $K_{2}$ is at most countable. It is easy to see that $K_{1} \cap S$ is also a Xiong chaotic set which is dense in $K_{1}$. By Theorem 4.3, $K_{1}$ is a weakly mixing set of order $n$.

Remark 4.5. It should be noticed that weakly mixing sets (of finite order $n$ ) are perfect. Hence, they are more restrained than Xiong chaotic sets, because any infinite subsets of Xiong chaotic sets (of finite order $n$ ) are also Xiong chaotic sets.

Let $(X, f)$ be a dynamical system with $x_{0} \in X, n \geq 2$ and $\delta>0$. Define

$$
D_{n}^{\delta}(X, f)=\left\{\left(x_{1}, x_{2}, \ldots, x_{n}\right) \in X^{n}: \limsup _{k \rightarrow \infty} \min _{1 \leq i<j \leq n} d\left(f^{k}\left(x_{i}\right), f^{k}\left(x_{j}\right)\right) \geq \delta\right\},
$$

and

$$
D_{n}^{\delta}(X, f)\left(x_{0}\right)=\left\{\left(x_{1}, \ldots, x_{n-1}\right) \in X^{n-1}:\left(x_{0}, x_{1}, \ldots, x_{n-1}\right) \in D_{n}^{\delta}(X, f)\right\} .
$$

Proposition 4.6. Let $(X, f)$ be a dynamical system and $A \subset X$ a weakly mixing set of order 2(n-1) with $n \geq 2$. Then there exists a $\delta>0$ such that, for every $x_{0} \in A$, $D_{n}^{\delta}(X, f)\left(x_{0}\right) \cap A^{n-1}$ is residual in $A^{n-1}$.

Proof. Since $A$ is perfect, we can choose a $\delta>0$ and 2(n-1) distinct points $u_{1,1}, u_{1,2}, \ldots, u_{n-1,1}, u_{n-1,2} \in A$ so that $d\left(u_{i_{1}, i_{2}}, u_{j_{1}, j_{2}}\right)>4 \delta$ for $\left(i_{1}, i_{2}\right) \neq\left(j_{1}, j_{2}\right)$. Fix $x_{0} \in A$. For every $\varepsilon>0$, set

$D_{\varepsilon}=\left\{\left(x_{1}, \ldots, x_{n-1}\right) \in X^{n-1}: \min _{0 \leq i<j \leq n-1} d\left(f^{k}\left(x_{i}\right), f^{k}\left(x_{j}\right)\right)>\delta-\varepsilon\right.$ for some $\left.k>\frac{1}{\varepsilon}\right\}$.

It is easy to verify that $D_{\varepsilon}$ is an open subset of $X^{n-1}$ and that

$$
D_{n}^{\delta}(X, f)\left(x_{0}\right)=\bigcap_{m=1}^{\infty} D_{\frac{1}{m}} .
$$

Therefore it is sufficient to prove that $D_{\varepsilon} \cap A^{n-1}$ is dense in $A^{n-1}$ for every $\varepsilon>0$.

Fix $\varepsilon>0$ and $n-1$ open subsets $U_{1}, \ldots, U_{n-1}$ of $X$ intersecting $A$. By Theorem 4.3, there is a Xiong chaotic set $S$ of order $2(n-1)$ which is dense in $A$. Observing that $A$ is perfect, for each $i=1, \ldots, n-1$ choose $y_{i, 1}, y_{i, 2} \in U_{i} \cap S$ with $y_{i, 1} \neq y_{i, 2}$. Define a map $g:\left\{y_{1,1}, y_{1,2}, \ldots, y_{n-1,1}, y_{n-1,2}\right\} \rightarrow A$ as $g\left(y_{i, j}\right)=u_{i, j}$ for $i=1, \ldots, n-1$ and $j=1,2$. Then there is an increasing subsequence $\left\{q_{l}\right\}_{l=1}^{\infty}$ in $\mathbb{N}$ such that $\lim _{l \rightarrow \infty} f^{q_{l}}\left(y_{i, j}\right)=g\left(y_{i, j}\right)=u_{i, j}$ for $i=1, \ldots, n-1$ and $j=1,2$. Pick $k>1 / \varepsilon$ such that $d\left(f^{k}\left(y_{i, j}\right), u_{i, j}\right)<\delta$ for $i=1, \ldots, n-1$ and $j=1,2$. There is at most one pair $\left(i_{0}, j_{0}\right)$ such that $d\left(f^{k}\left(x_{0}\right), u_{i_{0}, j_{0}}\right)<2 \delta$. For each $i=1, \ldots, n-1$, if $i \neq i_{0}$, let $x_{i}=y_{i, 1}$, and if $i=i_{0}$, let $x_{i}=y_{i_{0}}, \overline{j_{0}}$, where $\overline{j_{0}} \in\{1,2\}$ and $\overline{j_{0}} \neq j_{0}$. 
Then $x_{i} \in U_{i}$ for $i=1, \ldots, n-1$ and

$$
\min _{0 \leq i<j \leq n-1} d\left(f^{k}\left(x_{i}\right), f^{k}\left(x_{j}\right)\right)>\delta,
$$

which implies that $D_{\varepsilon} \cap A^{n-1}$ is dense in $A^{n-1}$.

\section{Weakly mixing sets}

5A. Weakly mixing sets. By Proposition 3.6, we have the following result.

Lemma 5.1. Let $(X, f)$ be a dynamical system and $A \subset X$ a nontrivial closed subset with $n \geq 2$. Then $A$ is weakly mixing for $f$ if and only if it is weakly mixing for $f^{n}$.

A dynamical system $(X, f)$ is called an $F$-system if it is totally transitive and has a dense set of periodic points [Furstenberg 1967]. It is shown in [Furstenberg 1967] that an $F$-system is disjoint from any minimal system. It is not hard to see that every $F$-system is weakly mixing (see [Banks 1997, Theorem 1.1]). We say a dynamical system $(X, f)$ has dense small periodic sets if for any nonempty open subset $U$ of $X$ there exists a nonempty closed subset $K$ of $U$ and a $k \in \mathbb{N}$ such that $f^{k}(K) \subset K$. A dynamical system $(X, f)$ is called an $H Y$-system if it is totally transitive and has dense small periodic sets. It is shown in [Huang and Ye 2005] that an $H Y$-system is weakly mixing and disjoint from any minimal system.

It is interesting when a totally transitive set or a weakly mixing set of finite order is also a weakly mixing set. Recall that a point $x \in X$ is distal provided that if $(x, y)$ is proximal and $y \in \overline{\operatorname{Orb}(x, f)}$ then $x=y$. The following fact is Corollary 11 from [Oprocha and Zhang 2013].

Theorem 5.2. Let $(X, f)$ be a dynamical system and $A \subset X$ a weakly mixing set of order 2. If the set of all distal points in $A$ is dense in A, then $A$ is weakly mixing of all orders.

We show that Theorem 5.2 can be generalized in the following way.

Theorem 5.3. Let $(X, f)$ be a dynamical system and $A \subset X$ a weakly mixing set of order 2. If for every open subset $U$ of $X$ intersecting $A$ there is a dynamical system $(Y, g)$ with a distal point $y \in Y$ and an open neighborhood $V \subset Y$ of $y$ and a point $x \in A \cap U$ such that $N(y, V) \subset N(x, U)$, then $A$ is weakly mixing of all orders.

Proof. By Theorem 3.1, it is sufficient to show that, for any $n \geq 2$ and any $n+1$ open subsets $U_{1}, V_{1}, V_{2}, \ldots, V_{n}$ of $X$ intersecting $A$,

$$
N\left(U_{1} \cap A, V_{1}\right) \cap \bigcap_{i=2}^{n} N\left(V_{i} \cap A, V_{i}\right) \neq \varnothing .
$$


By assumption, for $i=2, \ldots, n$ there are points $x_{i} \in V_{i} \cap A$ and distal points $y_{i}$ (in some dynamical systems) and their open neighborhoods $W_{i}$ such that we have $N\left(y_{i}, W_{i}\right) \subset N\left(x_{i}, V_{i}\right)$ and hence $\bigcap_{i=2}^{n} N\left(y_{i}, W_{i}\right) \subset \bigcap_{i=2}^{n} N\left(x_{i}, V_{i}\right)$. But the product of distal points is also distal, thus by [Furstenberg 1981] the following set intersects every IP-set:

$$
N\left(\left(y_{2}, \ldots, y_{n}\right), W_{2} \times \cdots \times W_{n}\right) \subset \bigcap_{i=2}^{n} N\left(y_{i}, W_{i}\right) \subset \bigcap_{i=2}^{n} N\left(x_{i}, V_{i}\right) .
$$

But, by Corollary 3.5, $N\left(U_{1} \cap A, V_{1}\right)$ contains an IP-set, which finishes the proof.

We say that a subset $A$ of $X$ has dense small periodic sets if, for any open subset $U$ of $X$ intersecting $A$, there exists a closed subset $K$ of $U$ intersecting $A$ and a $k \in \mathbb{N}$ such that $f^{k}(K) \subset K$. Then, observing that weak mixing of order 2 implies total transitivity, we have the following theorem.

Theorem 5.4. Let $(X, f)$ be a dynamical system and $A \subset X$ a nontrivial closed subset. If $A$ is totally transitive and has dense small periodic sets, then $A$ is weakly mixing.

Proof. First we show that $A$ is weakly mixing of order 2 . Let $U_{1}, V_{1}, V_{2}$ be open subsets of $X$ intersecting $A$. Since $A$ has dense small periodic sets, there exists a closed subset $K$ of $V_{2}$ intersecting $A$ and a $k \in \mathbb{N}$ such that $f^{k}(K) \subset K$. Since $A$ is transitive for $f^{k}$, there is an $m \in \mathbb{N}$ such that $m \in N_{f^{k}}\left(U_{1} \cap A, V_{1}\right)$. Then $k m \in N\left(U_{1} \cap A, V_{1}\right) \cap N\left(V_{2} \cap A, V_{2}\right)$, which implies that $A$ is weakly mixing of order 2 by Theorem 3.1 .

Now we show that $A$ satisfies the requirement of Theorem 5.3. Fix an open subset $U$ of $X$ intersecting $A$. There exists a closed subset $S$ of $U$ intersecting $A$ and a $k \in \mathbb{N}$ such that $f^{k}(S) \subset S$. Pick a point $x \in S \cap A$. Then $k \mathbb{N} \subset N(x, U)$. Let $Y=\{0,1, \ldots, k-1\}$ and $g: Y \rightarrow Y, g(i)=i+1(\bmod k)$. Let $y=0$ and $V=\{0\}$. Then $y$ is a distal point in $(Y, g)$ and $N(y, V)=k \mathbb{N} \subset N(x, U)$. Hence $A$ is weakly mixing of all orders by Theorem 5.3.

5B. Proximal relations. It is shown in [Akin and Kolyada 2003] that if $(X, f)$ is weakly mixing, then, for every $x \in X$, the set $\operatorname{Prox}_{2}(f)(x)$ is residual in $X$. In [Oprocha and Zhang 2013] it was proved that, for every weakly mixing set $A$ and every $x \in A$, the set $\operatorname{Prox}_{2}(f)(x) \cap A$ is residual in $A$. We will show that the same is true if we consider proximal tuples instead of pairs. First, we use a method of construction from [Oprocha and Zhang 2013, Lemma 16] to prove the following result.

Lemma 5.5. Let $(X, f)$ be a dynamical system and $A \subset X$ a weakly mixing set with $x \in A$. Then, for every $n \geq 2$, any open subsets $U_{1}, U_{2}, \ldots, U_{n}$ of $X$ intersecting $A$ and each $\varepsilon>0$, there are $a y_{i} \in U_{i} \cap A$ for $i=1,2, \ldots, n$ and an $m \in \mathbb{N}$ such that 
$\left(\right.$ with $\left.y_{0}=x\right)$

$$
\max _{0 \leq i<j \leq n} d\left(f^{m}\left(y_{i}\right), f^{m}\left(y_{j}\right)\right) \leq \varepsilon .
$$

Proof. Fix $n \geq 2$, open subsets $U_{1}, U_{2}, \ldots, U_{n}$ of $X$ intersecting $A$ and $\varepsilon>0$. Let $\left\{V_{1}, \ldots, V_{k}\right\}$ be a cover of $X$ consisting of open sets with diameters less than $\varepsilon / 2$.

There exists an $s_{1} \in\{1,2, \ldots, k\}$ with $x \in V_{s_{1}}$. By weak mixing of $A$, there exist an $m_{1}>0$ and an open set $U_{i}^{(1,1)} \subset U_{i}$ intersecting $A$ such that $f^{m_{1}}\left(U_{i}^{(1,1)}\right) \subset V_{s_{1}}$ for $i=1,2, \ldots, n$.

For some $q \geq 1$, construct open sets $U_{i}^{(q, 1)}, U_{i}^{(q, 2)}, \ldots, U_{i}^{(q, q)} \subset U_{i}$ intersecting $A$ for $i=1,2, \ldots, n$, pairwise distinct integers $s_{1}, s_{2}, \ldots, s_{q} \subset\{1,2, \ldots, k\}$, and an integer $m_{q}>0$ such that

$$
f^{m_{q}}\left(U_{i}^{(q, r)}\right) \subset V_{s_{r}} \text { for } r=1,2, \ldots, q, i=1,2, \ldots, n .
$$

If $f^{m_{q}}(x) \notin \bigcup_{r=1}^{q} V_{s_{r}}$, then we can choose $s_{q+1} \in\{1,2, \ldots, k\} \backslash\left\{s_{1}, s_{2}, \ldots, s_{q}\right\}$ and an open set $U^{(q, q+1)}$ containing $x$ (and intersecting $A$ ) so that $f^{m_{q}}\left(U^{(q, q+1)}\right) \subset V_{s_{q+1}}$. By weak mixing of $A$, there exist open sets $U_{i}^{(q+1,1)}, U_{i}^{(q+1,2)}, \ldots, U_{i}^{(q+1, q+1)} \subset U_{i}$ intersecting $A$ and a $p>0$ such that, for $r=1,2, \ldots, q+1$ and $i=1,2, \ldots, n$, we have $f^{p}\left(U_{i}^{(q+1, r)}\right) \subset U_{i}^{(q, r)}$, where $U_{i}^{(q, q+1)}=U^{(q, q+1)}$ for $i=1,2, \ldots, n$. Now if we set $m_{q+1}=m_{q}+p$, then for $r=1,2, \ldots, q+1$ and $i=1,2, \ldots, n$ we have

$$
f^{m_{q+1}}\left(U_{i}^{(q+1, r)}\right)=f^{m_{q}}\left(f^{p}\left(U_{i}^{(q+1, r)}\right)\right) \subset f^{m_{q}}\left(U_{i}^{(q, r)}\right) \subset V_{s_{r}} .
$$

Obviously, since $q \leq k$, we cannot extend the sequence $s_{1}, s_{2}, \ldots, s_{q}$ any further by the above procedure. Hence, we have that $f^{m_{q}}(x) \in \bigcup_{r=1}^{q} V_{s_{r}}$, and in particular $f^{m_{q}}(x) \in V_{s_{\ell}}$ for some $\ell \in\left\{s_{1}, \ldots, s_{q}\right\}$. But then by the construction we have $f^{m_{q}}\left(U_{i}^{(q, \ell)}\right) \subset V_{s_{\ell}}$ for $i=1,2, \ldots, n$, and so if we fix any $y_{i} \in U_{i}^{(q, \ell)} \cap A \subset U_{i} \cap A$ then $f^{m_{q}}\left(y_{i}\right) \in V_{s_{\ell}}$, finishing the proof.

Theorem 5.6. Let $(X, f)$ be a dynamical system and $A \subset X$ a weakly mixing set. Then, for every $x_{0} \in A$ and $n \geq 2$, the set $\operatorname{Prox}_{n}(f)\left(x_{0}\right) \cap A^{n-1}$ is residual in $A^{n-1}$. Proof. Fix any $x_{0} \in A$ and $n \geq 2$. For every $\varepsilon>0$, set $P_{\varepsilon}=\left\{\left(x_{1}, \ldots, x_{n-1}\right) \in X^{n-1}: \max _{0 \leq i<j \leq n-1} d\left(f^{k}\left(x_{i}\right), f^{k}\left(x_{j}\right)\right)<\varepsilon\right.$ for some $\left.k \geq 0\right\}$.

It is easy to verify that $P_{\varepsilon}$ is an open subset of $X^{n-1}$. By Lemma $5.5, P_{\varepsilon} \cap A^{n-1}$ is dense in $A^{n-1}$. This, by the fact that

$$
\operatorname{Prox}_{n}(f)\left(x_{0}\right)=\bigcap_{m=1}^{\infty} P_{\frac{1}{m}},
$$

proves that $\operatorname{Prox}_{n}(f)\left(x_{0}\right) \cap A^{n-1}$ is residual in $A^{n-1}$. 
Let $(X, f)$ be a dynamical system with $x_{0} \in X, n \geq 2$ and $\delta>0$. Define $L Y_{n}^{\delta}(X, f)=\left\{\left(x_{1}, x_{2}, \ldots, x_{n}\right) \in X^{n}:\left(x_{1}, \ldots, x_{n}\right)\right.$ is $n$-scrambled with modular $\left.\delta\right\}$, and

$$
L Y_{n}^{\delta}(X, f)\left(x_{0}\right)=\left\{\left(x_{1}, \ldots, x_{n-1}\right) \in X^{n-1}:\left(x_{0}, x_{1}, \ldots, x_{n-1}\right) \in L Y_{n}^{\delta}(X, f)\right\} .
$$

The following fact is a direct corollary of Proposition 4.6 and Theorem 5.6.

Theorem 5.7. Let $(X, f)$ be a dynamical system and $A \subset X$ a weakly mixing set. Then, for every $n \geq 2$, there exists $a \delta>0$ such that, for every $x_{0} \in A$, it holds that $L Y_{n}^{\delta}(X, f)\left(x_{0}\right) \cap A^{n-1}$ is residual in $A^{n-1}$.

5C. Local independent sets. Let $(X, f)$ be a dynamical system. Following [Kerr and Li 2007], for a tuple $\boldsymbol{A}=\left(A_{1}, A_{2}, \ldots, A_{k}\right)$ of subsets of $X$, we say that a nonempty subset $F \subset \mathbb{N}_{0}$ is an independence set for $\boldsymbol{A}$ if, for any nonempty finite subset $J \subset F$, we have

$$
\bigcap_{j \in J} f^{-j}\left(A_{s(j)}\right) \neq \varnothing
$$

for any $s \in\{1, \ldots, k\}^{J}$. We shall denote the collection of all independence sets for $\boldsymbol{A}$ by $\operatorname{Ind}\left(A_{1}, A_{2}, \ldots, A_{k}\right)$ or Ind $\boldsymbol{A}$. According to the best knowledge of the authors, the above notion of independence sets was first presented in [Huang and Ye 2006] under the name interpolating set (see also [Glasner and Weiss 1995]) and in [Huang 2006] when defining strong scrambled pairs. Later, the authors of [Huang et al. 2012] systematically studied independence sets in topological and measurable dynamics. In particular, they proved the following result (see [Huang et al. 2012, Theorem 5.1]).

Theorem 5.8. For a dynamical system $(X, f)$, the following conditions are equivalent:

(5.8.1) $(X, f)$ is weakly mixing.

(5.8.2) For any two nonempty open subsets $U_{1}, U_{2}$ of $X$, $\operatorname{Ind}\left(U_{1}, U_{2}\right)$ contains an infinite set.

(5.8.3) For any $n \in \mathbb{N}$ and any nonempty open subsets $U_{1}, U_{2}, \ldots, U_{n}$ of $X$, there is an $I P$-set in $\operatorname{Ind}\left(U_{1}, U_{2}, \ldots, U_{n}\right)$.

In the spirit of [Huang et al. 2012] we introduce a local definition of independence sets as follows.

Definition 5.9. Let $(X, f)$ be a dynamical system with $\varnothing \neq A \subset X$, and let $U_{1}, U_{2}, \ldots, U_{n}$ be open subsets of $X$ intersecting $A$. We say that a nonempty 
subset $F \subset \mathbb{N}_{0}$ is an independence set for $\left(U_{1}, U_{2}, \ldots, U_{n}\right)$ with respect to $A$ if, for every nonempty finite subset $J \subset F$ and $s \in\{1,2, \ldots, n\}^{J}$,

$$
\bigcap_{j \in J} f^{-j}\left(U_{s(j)}\right)
$$

is a nonempty open subset of $X$ intersecting $A$.

Now we can employ this definition to state a theorem analogous to Theorem 5.8. Theorem 5.10. Let $(X, f)$ be a dynamical system and $A \subset X$ a nontrivial closed set. Then the following conditions are equivalent:

(5.10.1) A is a weakly mixing set.

(5.10.2) For every $n \geq 2$ and any open subsets $U_{1}, U_{2}, \ldots, U_{n}$ of $X$ intersecting $A$, there exists a $t \in \mathbb{N}$ such that $\{0, t\}$ is an independence set for $\left(U_{1}, U_{2}, \ldots, U_{n}\right)$ with respect to $A$.

(5.10.3) For every $n \geq 2$ and any open subsets $U_{1}, U_{2}, \ldots, U_{n}$ of $X$ intersecting $A$, there exists a sequence $\left\{t_{j}\right\}_{j=1}^{\infty}$ in $\mathbb{N}$ such that $\{0\} \cup F S\left(\left\{t_{j}\right\}_{j=1}^{\infty}\right)$ is an independence set for $\left(U_{1}, U_{2}, \ldots, U_{n}\right)$ with respect to $A$.

Proof. (5.10.2) $\Rightarrow$ (5.10.1) Fix $n \geq 2$ and fix open subsets $U_{1}, U_{2}, \ldots, U_{n}$, $V_{1}, V_{2}, \ldots, V_{n}$ of $X$ intersecting $A$. By assumption there exists a $t \geq 1$ such that $\{0, t\}$ is an independence set for $\left(U_{1}, U_{2}, \ldots, U_{n}, V_{1}, V_{2}, \ldots, V_{n}\right)$ with respect to $A$. For $i=1,2, \ldots, n$, we have that $U_{i} \cap f^{-t}\left(V_{i}\right)$ is a nonempty open subset of $X$ intersecting $A$. Therefore, $t \in \bigcap_{i=1}^{n} N\left(U_{i} \cap A, V_{i}\right)$, which implies that $A$ is weakly mixing of order $n$.

(5.10.1) $\Rightarrow(5.10 .3)$ Let $U_{1}, U_{2}, \ldots, U_{n}$ be open subsets of $X$ intersecting $A$. First, there exists a $t_{1} \in \mathbb{N}$ such that

$$
t_{1} \in \bigcap_{i_{1}, i_{2} \in\{1,2, \ldots, n\}} N\left(U_{i_{1}} \cap A, U_{i_{2}}\right) .
$$

That is, for every $i_{1}, i_{2} \in\{1,2, \ldots, n\}$, we have that $U_{i_{1}} \cap f^{-t_{1}}\left(U_{i_{2}}\right)$ is a nonempty open set intersecting $A$. Therefore, there exists a $t_{2} \in \mathbb{N}$ such that

$$
t_{2} \in \bigcap_{i_{1}, i_{2}, i_{3}, i_{4} \in\{1,2, \ldots, n\}} N\left(U_{i_{1}} \cap f^{-t_{1}}\left(U_{i_{2}}\right) \cap A, U_{i_{3}} \cap f^{-t_{1}}\left(U_{i_{4}}\right)\right) .
$$

That is, for every $i_{1}, i_{2}, i_{3}, i_{4} \in\{1,2, \ldots, n\}$,

$$
U_{i_{1}} \cap f^{-t_{1}}\left(U_{i_{2}}\right) \cap f^{-t_{2}} U_{i_{3}} \cap f^{-\left(t_{1}+t_{2}\right)}\left(U_{i_{4}}\right)
$$

is a nonempty open set of $X$ intersecting $A$. Then $\left\{0, t_{1}, t_{2}, t_{1}+t_{2}\right\}$ is an independent set of $\left(U_{1}, U_{2}, \ldots, U_{n}\right)$ with respect to $A$ and the result follows by induction.

$(5.10 .3) \Rightarrow(5.10 .2)$ The implication is trivial. 
Remark 5.11. Let $\boldsymbol{A}=\left(A_{1}, \ldots, A_{k}\right)$ be a tuple of subsets of $X$. If $F$ is an independence set for $\boldsymbol{A}$, then for every $m \in \mathbb{N}_{0}$ the subset $F-m$ defined by $\{n-m: n \geq m$ and $n \in F\}$ is also an independence set for $\boldsymbol{A}$. So we may also assume that an independence set of $\boldsymbol{A}$ contains 0. But in Theorem 5.10 we cannot replace $\{0\} \cup F S\left(\left\{t_{j}\right\}_{j=1}^{\infty}\right)$ by $F S\left(\left\{t_{j}\right\}_{j=1}^{\infty}\right)$, as shown by the following example.

Example 5.12. Consider $X=\Sigma_{3}^{+}=\{0,1,2\}^{\mathbb{N}_{0}}$ and define

$$
A=\{1,2\}^{\mathbb{N}_{0}} \cup C_{X}[00] \text {. }
$$

For any open subsets $U_{1}, \ldots, U_{n}$ of $X$ intersecting $A$, we can easily define words $w^{(1)}, \ldots, w^{(n)}$ of the same length, $M \geq 2$, (with symbols in the alphabet $\{0,1,2\}$ ) such that $C\left[w^{(i)}\right] \subset A$ for all $i=1, \ldots, n$. This implies that the set $J=\{k M: k \in \mathbb{N}\}$ is an independence set for $\left(U_{1}, U_{2}, \ldots, U_{n}\right)$ with respect to $A$. But $A$ is not weakly mixing of order 2 , because, for example, $N\left(A \cap C_{X}[1], C_{X}[0]\right)=\varnothing$.

Question. Let $(X, f)$ be a dynamical system and $A \subset X$ a weakly mixing set of order 2. Is it true that for every two open subsets $U_{1}, U_{2}$ of $X$ intersecting $A$, there exists a $t \in \mathbb{N}$ such that $\{0, t\}$ is an independence set for $\left(U_{1}, U_{2}\right)$ with respect to $A$ ?

\section{Topological graphs}

For any integer $n \geq 2$, it is known that a weakly mixing set of order $n$ does not have to be weakly mixing of order $n+1$; even worse, it may happen that there is no weakly mixing set of order $n+1$ in a system with weakly mixing sets of order $n$ [Oprocha and Zhang 2014]. Note that the examples in [Oprocha and Zhang 2014] are subshifts, and for every dynamical system on the unit interval $([0,1], f)$ with positive topological entropy there is an $m>0$ and a closed set $\Lambda$ invariant for $f^{m}$ such that $\left(\Lambda, f^{m}\right)$ is conjugated with the full shift on two symbols. In particular, in every interval map with weakly mixing sets we can find sets which are weakly mixing of order $n$ but not $n+1$. However, in [Oprocha and Zhang 2011] the authors proved that on the unit interval every weakly mixing set of order 2 is arbitrarily close (in the Hausdorff metric) to a weakly mixing set of all orders. So even if these sets are not the same, they are arbitrarily close to each other. Theorem 6.1 completes our research on weakly mixing sets in dimension one, showing that the above fact also holds for all topological graphs.

Theorem 6.1. Let $(G, f)$ be a dynamical system acting on the topological graph $G$ and let $A \subset G$ be a weakly mixing subset of order 2 . Then for every $\varepsilon>0$ there is a weakly mixing subset $D \subset G$ such that $\mathcal{H}_{d}(A, D) \leq \varepsilon$, where $\mathcal{H}_{d}(A, D)$ denotes the Hausdorff distance between $A$ and D. 
Proof. Let $\varepsilon>0$. Pick nonempty open subsets $U_{1}, \ldots, U_{s}$ of $G$ with diameters at most $\varepsilon$ such that $A \subset \bigcup_{i=1}^{s} U_{i}$ and $A \cap U_{i} \neq \varnothing$ for $i=1, \ldots, s$. By Lemma 2.5 the set $A$ is perfect; therefore, for every $i=1, \ldots, s$ it is possible to select an open set $V_{i} \subset U_{i}$ contained in the interior of an edge of $G$ such that $V_{i} \cap A \neq \varnothing$ and $V_{1}, \ldots, V_{s}$ are pairwise disjoint.

Claim. For every $i=1, \ldots, s$ there is an interval $I_{i} \subset V_{i}$, its disjoint closed subintervals $K_{2 i}, K_{2 i+1}$ and an integer $n_{i}>0$ such that

(6.1.1) $K_{2 i}, K_{2 i+1}$ form a strong 2-horseshoe for $f^{n_{i}}$; that is, $K_{p} \stackrel{f^{n_{i}}}{\Longrightarrow} K_{q}$ for all $p, q \in\{2 i, 2 i+1\}$, and

(6.1.2) both sets $\operatorname{int}\left(K_{2 i}\right), \operatorname{int}\left(K_{2 i+1}\right)$ as well as every connected component of the set $I_{i} \backslash\left(K_{2 i} \cup K_{2 i+1}\right)$ intersect $A$.

Proof of Claim. Let $I_{i}$ be any closed interval contained in $V_{i}$ such that $\operatorname{int}\left(I_{i}\right) \cap A \neq \varnothing$. Let us identify $I_{i}$ with $[0,1]$. Observe that $A$ is a weakly mixing set of order 2, and so it contains no isolated points. Thus there are points $0=a_{0}<a_{1}<\cdots<a_{6}<a_{7}=1$ in $I_{i}$ such that $\left(a_{j}, a_{j+1}\right) \cap A \neq \varnothing$ for all $j=0, \ldots, 6$. Define $I_{i, j}=\left[a_{2 j}, a_{2 j+1}\right]$ for $j=0,1,2,3$. Since $A$ is weakly mixing of order 2 , there are $k>0, r>0$ such that

$$
\begin{array}{ll}
f^{r}\left(I_{i, 1}\right) \cap\left(a_{1}, a_{2}\right) \neq \varnothing, & f^{k}\left(I_{i, 2}\right) \cap\left(a_{1}, a_{2}\right) \neq \varnothing, \\
f^{r}\left(I_{i, 1}\right) \cap\left(a_{5}, a_{6}\right) \neq \varnothing, & f^{k}\left(I_{i, 2}\right) \cap\left(a_{5}, a_{6}\right) \neq \varnothing .
\end{array}
$$

If $I_{i, 1} \stackrel{f^{r}}{\Longrightarrow} I_{i, 1}, I_{i, 1} \stackrel{f^{r}}{\Longrightarrow} I_{i, 2}$ and $I_{i, 2} \stackrel{f^{k}}{\Longrightarrow} I_{i, 1}, I_{i, 2} \stackrel{f^{k}}{\Longrightarrow} I_{i, 2}$ then by Lemma 2.7 the intervals $I_{i, 1}$ and $I_{i, 2}$ form a 2-horseshoe for $f^{k+r}$.

Otherwise there are $p \in\{1,2\}$ and $j \in\{k, r\}$ such that $I_{i, p} \stackrel{f^{j}}{\Longrightarrow} I_{i, 0}$ and $I_{i, p} \stackrel{f^{j}}{\Longrightarrow} I_{i, 3}$. Next, if we consider $I_{i, 0}$, then there is an $l>0$ such that $I_{i, 0} \stackrel{f^{l}}{\Longrightarrow} I_{i, 0}$ and $I_{i, 0} \stackrel{f^{l}}{\Longrightarrow} I_{i, 3}$, or we have the second possibility that $I_{i, 0} \stackrel{f^{l}}{\Longrightarrow} I_{i, 1}$ and $I_{i, 0} \stackrel{f^{l}}{\Longrightarrow} I_{i, 2}$ which implies that $I_{i, 0} \stackrel{f^{l+j}}{\Longrightarrow} I_{i, 0}$ and $I_{i, 0} \stackrel{f^{l+j}}{\Longrightarrow} I_{i, 3}$ again by applying Lemma 2.7 . We can repeat the same arguments for $I_{i, 3}$, and with the help of Lemma 2.7 finally obtain that $I_{i, 0}, I_{i, 3}$ form a 2-horseshoe for some iterate of $f$.

Now for each $i=1, \ldots, s$ let sets $K_{2 i}, K_{2 i+1}$ be provided for $V_{i}$ by the claim, and let $J_{i}$ be the connected component of $I_{i} \backslash\left(K_{2 i} \cup K_{2 i+1}\right)$ such that $K_{2 i}$ and $K_{2 i+1}$ are contained in different connected components of $I_{i} \backslash J_{i}$. We prove by induction that for every $m=1, \ldots, s$ intervals $K_{2}, \ldots, K_{2 m+1}$ form a horseshoe for some iterate $f^{h}, h \in \mathbb{N}$; that is, $K_{p} \stackrel{f^{h}}{\Longrightarrow} K_{q}$ for all $p, q \in\{2, \ldots, 2 m+1\}$.

By the construction we have proved the above statement for $m=1$, so we may assume that it holds for some $1 \leq m<s$. Therefore, there is a $t_{1}>0$ such that $K_{p} \stackrel{f^{t_{1}}}{\Longrightarrow} K_{q}$ for all $p, q \in\{2, \ldots, 2 m+1\}$ and a $t_{2}>0$ such that $K_{2 m+2}, K_{2 m+3}$ form a 2-horseshoe for $f^{t_{2}}$. If we set $t=t_{1} t_{2}$, then we have that $K_{2}, \ldots, K_{2 m+1}$ form a horseshoe for $f^{t}$, and that $K_{2 m+2}, K_{2 m+3}$ form a 2-horseshoe for $f^{t}$. Since 
$A$ is weakly mixing of order 2 , there are $k, r>0$ such that

$$
\begin{gathered}
f^{r}\left(K_{2}\right) \cap J_{1} \neq \varnothing, \quad f^{r}\left(K_{2}\right) \cap J_{m+1} \neq \varnothing, \\
f^{k}\left(K_{2 m+3}\right) \cap J_{1} \neq \varnothing, \quad f^{k}\left(K_{2 m+3}\right) \cap J_{m+1} \neq \varnothing .
\end{gathered}
$$

From the construction and the first two conditions we see that $K_{2} \stackrel{f^{r}}{\Longrightarrow} K_{2}$ or $K_{2} \stackrel{f^{r}}{\Longrightarrow} K_{3}$ and at the same time $K_{2} \stackrel{f^{r}}{\Longrightarrow} K_{2 m+2}$ or $K_{2} \stackrel{f^{r}}{\Longrightarrow} K_{2 m+3}$, which implies that $K_{2} \stackrel{f^{r+t}}{\Longrightarrow} K_{q}$ for every $q=2, \ldots, 2 m+3$ by Lemma 2.7. By a symmetric argument, we see that $K_{2 m+3} \stackrel{f^{k+t}}{\Longrightarrow} K_{q}$ for every $q=2, \ldots, 2 m+3$. Now applying Lemma 2.7 it is easy to verify that $K_{p} \stackrel{f^{r+k+3 t}}{\Longrightarrow} K_{q}$ for every $p, q \in\{2, \ldots, 2 m+3\}$. This completes the induction.

Since $K_{i}, i=2, \ldots, 2 s+1$, form a horseshoe, rewriting arguments in the proof of [Moothathu 2011, Theorem 9] (stated there for horseshoes in interval maps) we obtain that there is an $n>0$, an $f^{n}$-invariant closed subset $\Gamma \subset \bigcup_{i=1}^{s} V_{i}$ and a topological conjugacy $\pi:\left(\Gamma, f^{n}\right) \rightarrow\left(\Sigma_{s}, \sigma\right)$ between dynamical systems such that $\Gamma \cap V_{i} \neq \varnothing$ for each $i=1, \ldots, s$ (and hence $\mathcal{H}_{d}(A, \Gamma) \leq \varepsilon$ ), where $\left(\Sigma_{s}, \sigma\right)$ is the full shift over the alphabet $\{1, \ldots, s\}$. In particular $f^{n}$ is mixing on $\Gamma$, so indeed $\Gamma$ is a weakly mixing subset, which completes the proof.

\section{Acknowledgements}

The authors would like to thank the referee for useful comments that resulted in substantial improvements to this paper.

The first author was supported in part by National Natural Science Foundation of China, grant numbers 11401362 and 11471125 . The second author was supported by the Polish Ministry of Science and Higher Education from sources for science in the years 2013-2014, grant number IP2012 004272. The third author was supported by Foundation for the Author of National Excellent Doctoral Dissertation of China, grant number 201018 and National Natural Science Foundation of China, grant number 11271078.

\section{Appendix}

Proof of Example 3.2. Let us endow [0,1] with the Euclidean metric. Take any increasing sequences $\left\{a_{i}\right\}_{i \in \mathbb{Z}},\left\{b_{i}\right\}_{i \in \mathbb{Z}} \subset \mathbb{R} \backslash \mathbb{Q}$ such that $1 / 2<a_{-1}<a_{0}<b_{0}<b_{1}<a_{1}$ and $\lim _{i \rightarrow \infty} a_{-i}=\lim _{i \rightarrow \infty} b_{-i}=0$ and $\lim _{i \rightarrow \infty} a_{i}=\lim _{i \rightarrow \infty} b_{i}=1$. Furthermore, we assume that every interval $\left(a_{i}, a_{i+1}\right)$ contains at most one element of the set $\left\{2^{-k}: k \in \mathbb{N}\right\}$.

We will define homeomorphisms $F_{i}:[0,1] \rightarrow[0,1]$ for $i=0, \ldots, 8$. Define $F_{0}=$ id and set $F_{i}(0)=0$ and $F_{i}(1)=1$ for every $i$. For each $i \in \mathbb{Z}$ we set $F_{1}\left(a_{i}\right)=a_{i+1}$ and $F_{3}\left(b_{i}\right)=b_{i+1}$. On each interval $\left[b_{i}, b_{i+1}\right]$ we define $F_{3}$ as a 
linear map, which completes the definition of $F_{3}$, since values of $F_{3}$ at endpoints of every such interval have already been set. For $i \geq 0$ we define $F_{1}$ on $\left[a_{i}, a_{i+1}\right]$ as a linear map. Now, fix any sequence of distinct points $\left\{c_{k}\right\}_{k=1}^{\infty} \subset\left(a_{0}, a_{1}\right)$ in such a way that $\left\{\overline{c_{k}: k \in \mathbb{N}}\right\}=\left[a_{0}, a_{1}\right]$. We are ready to define $F_{1}$ on the intervals $\left[a_{i}, a_{i+1}\right]$ for $i<0$. Suppose that $\left.F_{1}\right|_{\left[a_{-n}, 1\right]}$ is already defined for some $n \geq 0$. If $\left\{2^{-k}: k \in \mathbb{N}\right\} \cap\left[a_{-n-1}, a_{-n}\right]=\varnothing$ then we define $F_{1}$ as a linear map on $\left[a_{-n-1}, a_{-n}\right]$, and as a result $F_{1}$ is well-defined on the interval $\left[a_{-n-1}, 1\right]$. Otherwise there is a $k>0$ such that $2^{-k} \in\left(a_{-n-1}, a_{-n}\right)$ (hence $n \geq 1$ ). Define

$$
G:\left.\left.\left[a_{-n}, a_{-n+1}\right] \ni x \mapsto F_{1}\right|_{\left[a_{-1}, a_{0}\right]} \circ \cdots \circ F_{1}\right|_{\left[a_{-n}, a_{-n+1}\right]}(x) \in\left[a_{0}, a_{1}\right]
$$

and observe that there is a $q \in\left[a_{-n}, a_{-n+1}\right]$ such that $G(q)=c_{k}$. Now, we set $F_{1}\left(2^{-k}\right)=q$ and define $F_{1}$ to be linear on each of the intervals $\left[a_{-n-1}, 2^{-k}\right]$ and $\left[2^{-k}, a_{-n}\right]$. Then in this case $F_{1}$ is also well-defined on $\left[a_{-n-1}, 1\right]$. Induction completes the construction. Define inverses $F_{2}=F_{1}^{-1}$ and $F_{4}=F_{3}^{-1}$. Then for every $k$ there is an $n>0$ such that $F_{2}^{n}\left(c_{k}\right)=F_{1}^{-n}\left(c_{k}\right)=2^{-k}$.

We define $F_{5}(x)=1 / 2+1 / 2(2 x-1)^{3}$ and $F_{6}=F_{5}^{-1}$. Finally $F_{7}\left(2^{-k-1}\right)=2^{-k}$ for $k \in \mathbb{N}, F_{7}(1 / 2)=a_{1}$ and $F_{7}\left(a_{i}\right)=a_{i+1}$ for $i \in \mathbb{N}$. Between any two consecutive points in the set $\bigcup_{k \in \mathbb{N}}\left\{2^{-k}, a_{k}\right\}$ the map $F_{7}$ is linear, which again gives a welldefined homeomorphism. As the last map we take $F_{8}=F_{7}^{-1}$. Observe that for any $\delta, \varepsilon \in(0,1 / 2)$ there is an $n>0$ such that $F_{6}^{n}([1 / 2-\delta, 1 / 2+\delta]) \supset(\varepsilon, 1-\varepsilon)$.

Let $X=\Sigma_{9}^{+} \times[0,1]$ (endowed with the product metric given by the maximum distance on each coordinate) where $\Sigma_{9}^{+}=\{0,1, \ldots, 8\}^{\mathbb{N}_{0}}$ and let $T: X \rightarrow X$ be defined by

$$
T(\omega, x)=\left(\sigma(\omega), F_{\omega_{0}}(x)\right)
$$

with $\sigma$ the standard shift transformation on $\Sigma_{9}^{+}$. Thus $X$ is compact and $T$ is continuous.

For any symbol $a \in\{0,1, \ldots, 8\}$ and pairs $(0,0),(1,2),(3,4),(5,6),(7,8)$, let $\bar{a}$ be the replacement of $a$ by the second element of the respective pair. For example, $\overline{8}=7$. We extend this definition to words, putting $\overline{w_{0} \cdots w_{n}}=\overline{w_{n}} \cdots \overline{w_{0}}$. If, for a finite sequence $w$ of symbols in $\{0,1, \ldots, 8\}$, we denote by $F_{w}$ the composition $F_{w}=F_{w_{|w|-1}} \circ \cdots \circ F_{w_{1}} \circ F_{w_{0}}$, then $F_{w \bar{w}}=F_{\bar{w}} \circ F_{w}=$ id.

Before proceeding with the construction of a set $A$, let us make a few observations on these maps $F_{i}$. Fix any nonempty open sets $U, V$ with $\bar{U}, \bar{V} \subset(0,1)$. First of all $U \cap\left(b_{i}, b_{i+1}\right) \neq \varnothing$ for some $i \in \mathbb{Z}$, and hence there is a word $u$ consisting only of symbols 0,3 or 4 and such that

$$
F_{u}(U) \cap\left(a_{0}, a_{1}\right) \supset F_{u}(U) \cap\left(b_{0}, b_{1}\right) \neq \varnothing .
$$

In particular, there is a $k \in \mathbb{N}$ such that $c_{k} \in F_{u}(U)$. But then there is also an $s>0$ such that if we set $v=2^{s} 7^{k-1}$ (i.e., $v$ is a concatenation of $s$ repetitions of symbol 2 
and $k-1$ repetitions of symbol 7) then $F_{v}\left(c_{k}\right)=F_{7}^{k-1}\left(F_{2}^{s}\left(c_{k}\right)\right)=F_{7}^{k-1}\left(2^{-k}\right)=1 / 2$. In particular, there is a $\delta>0$ such that $[1 / 2-\delta, 1 / 2+\delta] \subset F_{u v}(U)$, where as usual $u v=u_{0} u_{1} \cdots u_{|u|-1} v_{0} v_{1} \cdots v_{|v|-1}$. But then there are an $\varepsilon>0$ and $m>0$ such that

$$
F_{w}([1 / 2-\delta, 1 / 2+\delta]) \supset(\varepsilon, 1-\varepsilon) \supset U \cup V
$$

if we set $w=6^{m}$. We have just shown that for any nonempty open sets $U, V$ with $\bar{U}, \bar{V} \subset(0,1)$ there are words $u, v, w$ such that

$$
F_{u v w}(U) \supset U \cup V .
$$

Now we are ready to construct set $A$. Let $\left\{\omega_{i}\right\}_{i=1}^{\infty}$ be any sequence containing all possible words (finite sequences) over the symbols $0,1, \ldots, 8$. Let

$$
\xi=\omega_{1} \bar{\omega}_{1} \omega_{2} \bar{\omega}_{2} \cdots \omega_{n} \bar{\omega}_{n} \cdots \in \Sigma_{9}^{+} \quad \text { and } \quad A=\{\xi\} \times[0,1] .
$$

Take any nonempty open sets $\tilde{U}, \tilde{V}$ intersecting $A$. Then there are an $i>0$ and open intervals $U, V$ such that $\bar{U}, \bar{V} \subset(0,1)$ and

$$
C\left[\omega_{1} \bar{\omega}_{1} \cdots \omega_{i} \bar{\omega}_{i}\right] \times U \subset \tilde{U} \quad \text { and } \quad C\left[\omega_{1} \bar{\omega}_{1} \cdots \omega_{i} \bar{\omega}_{i}\right] \times V \subset \tilde{V}
$$

Let words $u, v, w$ be provided for $U$ and $V$ by (2). By the definition, there is a $j>1$ such that $\omega_{j}=u v w \omega_{1} \bar{\omega}_{1} \cdots \omega_{i} \bar{\omega}_{i}$. Define $t=\sum_{r=1}^{j-1} 2\left|\omega_{r}\right|$ and $p=t+|u v w|$. Note that

$$
\begin{aligned}
T^{t}(\{\xi\} \times U) & =\left\{\sigma^{t}(\xi)\right\} \times F_{\omega_{1} \bar{\omega}_{1} \cdots \omega_{j-1} \bar{\omega}_{j-1}}(U)=\left\{\sigma^{t}(\xi)\right\} \times U \\
& =\left\{\omega_{j} \bar{\omega}_{j} \cdots\right\} \times U=\left\{u v w \omega_{1} \bar{\omega}_{1} \omega_{2} \bar{\omega}_{2} \cdots \omega_{i} \bar{\omega}_{i} \cdots\right\} \times U
\end{aligned}
$$

and therefore

$$
\begin{aligned}
T^{p}(\tilde{U} \cap A) & \supset T^{p}(\{\xi\} \times U) \supset\left\{\omega_{1} \bar{\omega}_{1} \cdots \omega_{i} \bar{\omega}_{i} \cdots\right\} \times F_{u v w}(U) \\
& \supset\left\{\omega_{1} \bar{\omega}_{1} \cdots \omega_{i} \bar{\omega}_{i} \cdots\right\} \times(U \cup V) .
\end{aligned}
$$

We have just shown that $p \in N(\tilde{U} \cap A, \tilde{U}) \cap N(\tilde{U} \cap A, \tilde{V})$.

Similarly, if in the above calculations $j$ was such that $\omega_{j}=\overline{u v w} \omega_{1} \bar{\omega}_{1} \cdots \omega_{i} \bar{\omega}_{i}$ then, since we have $F_{\overline{u v w}}(U \cup V)=F_{u v w}^{-1}(U \cap V) \subset U$ by (2), we obtain that $p \in N(\tilde{U} \cap A, \tilde{U}) \cap N(\tilde{V} \cap A, \tilde{U})$, as

$$
\begin{aligned}
& T^{p}(\tilde{U} \cap A) \supset\left\{\omega_{1} \bar{\omega}_{1} \cdots \omega_{i} \bar{\omega}_{i} \cdots\right\} \times F_{\overline{u v w}}(U) \subset \tilde{U}, \\
& T^{p}(\tilde{V} \cap A) \supset\left\{\omega_{1} \bar{\omega}_{1} \cdots \omega_{i} \bar{\omega}_{i} \cdots\right\} \times F_{\overline{u v w}}(V) \subset \tilde{U} .
\end{aligned}
$$

Finally, observe that each map $F_{i}$ preserves the ordering of $[0,1]$. Set $U=(a, b)$ and $V=(c, d)$ where $b<c$. If for some word $w$ we have $F_{w}(V) \cap U \neq \varnothing$, then $F_{w}(U) \subset[0, b)$, and in particular $F_{w}(U) \cap V=\varnothing$. Therefore, if we set $\tilde{U}=\Sigma_{9}^{+} \times U$ and $\tilde{V}=\Sigma_{9}^{+} \times V$, both intersecting $A$, then $N(\tilde{U} \cap A, \tilde{V}) \cap N(\tilde{V} \cap A, \tilde{U})=\varnothing$. This shows that $A$ is not a weakly mixing set of order 2, completing the proof. 


\section{References}

[Akin 2004] E. Akin, "Lectures on Cantor and Mycielski sets for dynamical systems", pp. 21-79 in Chapel Hill Ergodic Theory Workshops, edited by I. Assani, Contemp. Math. 356, Amer. Math. Soc., Providence, RI, 2004. MR 2005e:37018 Zbl 1064.37015

[Akin and Kolyada 2003] E. Akin and S. Kolyada, "Li-Yorke sensitivity", Nonlinearity 16:4 (2003), 1421-1433. MR 2004c:37016 Zbl 1045.37004

[Alsedà et al. 2003] L. Alsedà, M. A. del Río, and J. A. Rodríguez, "Transitivity and dense periodicity for graph maps”, J. Difference Equ. Appl. 9:6 (2003), 577-598. MR 2004d:37061 Zbl 1032.37025

[Banks 1997] J. Banks, "Regular periodic decompositions for topologically transitive maps", Ergodic Theory Dynam. Systems 17:3 (1997), 505-529. MR 98d:54074 Zbl 0921.54029

[Banks 1999] J. Banks, "Topological mapping properties defined by digraphs", Discrete Contin. Dynam. Systems 5:1 (1999), 83-92. MR 99j:54038 Zbl 0957.54020

[Blanchard and Huang 2008] F. Blanchard and W. Huang, "Entropy sets, weakly mixing sets and entropy capacity”, Discrete Contin. Dyn. Syst. 20:2 (2008), 275-311. MR 2009a:37026 Zbl 1151.37019

[Furstenberg 1967] H. Furstenberg, "Disjointness in ergodic theory, minimal sets, and a problem in Diophantine approximation”, Math. Systems Theory 1 (1967), 1-49. MR 35 \#4369 Zbl 0146.28502

[Furstenberg 1981] H. Furstenberg, Recurrence in ergodic theory and combinatorial number theory, Princeton University Press, 1981. MR 82j:28010 Zbl 0459.28023

[Glasner and Weiss 1995] E. Glasner and B. Weiss, "Quasi-factors of zero-entropy systems", J. Amer. Math. Soc. 8:3 (1995), 665-686. MR 95i:54048 Zbl 0846.28009

[Huang 2006] W. Huang, "Tame systems and scrambled pairs under an abelian group action", Ergodic Theory Dynam. Systems 26:5 (2006), 1549-1567. MR 2007j:37012 Zbl 1122.37009

[Huang and Ye 2005] W. Huang and X. Ye, "Dynamical systems disjoint from any minimal system", Trans. Amer. Math. Soc. 357:2 (2005), 669-694. MR 2005g:37012 Zbl 1072.37011

[Huang and Ye 2006] W. Huang and X. Ye, "A local variational relation and applications", Israel J. Math. 151 (2006), 237-279. MR 2006k:37033 Zbl 1122.37013

[Huang et al. 2004] W. Huang, S. Shao, and X. Ye, "Mixing and proximal cells along sequences", Nonlinearity 17:4 (2004), 1245-1260. MR 2005b:37015 Zbl 1055.37014

[Huang et al. 2012] W. Huang, H. Li, and X. Ye, "Family independence for topological and measurable dynamics", Trans. Amer. Math. Soc. 364:10 (2012), 5209-5242. MR 2931327 Zbl 1286.37017

[Kerr and Li 2007] D. Kerr and H. Li, "Independence in topological and $C^{*}$-dynamics", Math. Ann. 338:4 (2007), 869-926. MR 2009a:46126 Zbl 1131.46046

[Li 2011] J. Li, “Transitive points via Furstenberg family”, Topology Appl. 158:16 (2011), 2221-2231. MR 2012m:37012 Zbl 1234.37034

[Moothathu 2011] T. K. S. Moothathu, "Syndetically proximal pairs", J. Math. Anal. Appl. 379:2 (2011), 656-663. MR 2012c:37025 Zbl 1225.54011

[Mycielski 1964] J. Mycielski, "Independent sets in topological algebras", Fund. Math. 55 (1964), 139-147. MR 30 \#3855 Zbl 0124.01301

[Oprocha and Zhang 2011] P. Oprocha and G. Zhang, "On local aspects of topological weak mixing in dimension one and beyond”, Studia Math. 202:3 (2011), 261-288. MR 2012e:37011 Zbl 1217.37012

[Oprocha and Zhang 2012] P. Oprocha and G. Zhang, "On sets with recurrence properties, their topological structure and entropy”, Topology Appl. 159:7 (2012), 1767-1777. MR 2904065 Zbl 1244.37013 
[Oprocha and Zhang 2013] P. Oprocha and G. Zhang, "On weak product recurrence and synchronization of return times", Adv. Math. 244 (2013), 395-412. MR 3077877 Zbl 1286.37015

[Oprocha and Zhang 2014] P. Oprocha and G. Zhang, "On local aspects of topological weak mixing, sequence entropy and chaos", Ergodic Theory Dynam. Systems 34:5 (2014), 1615-1639. MR 3255435 Zbl 06366533

[Petersen 1970] K. E. Petersen, "Disjointness and weak mixing of minimal sets", Proc. Amer. Math. Soc. 24 (1970), 278-280. MR 40 \#3522 Zbl 0188.55503

[Xiong 2005] J. Xiong, "Chaos in a topologically transitive system", Sci. China Ser. A 48:7 (2005), 929-939. MR 2006g:37022 Zbl 1096.37018

[Xiong and Yang 1991] J. C. Xiong and Z. G. Yang, "Chaos caused by a topologically mixing map", pp. 550-572 in Dynamical systems and related topics (Nagoya, 1990), edited by K. Shiraiwa, Adv. Ser. Dynam. Systems 9, World Sci. Publ., River Edge, NJ, 1991. MR 93c:58153

[Ye and Zhang 2008] X. Ye and R. Zhang, "On sensitive sets in topological dynamics", Nonlinearity 21:7 (2008), 1601-1620. MR 2009j:37017 Zbl 1153.37322

Received November 27, 2013. Revised December 10, 2014.

JIAN LI

DEPARTMENT OF MATHEMATICS

SHANTOU UNIVERSITY

DAXUE RoAd No. 243

Shantou, GUANGDONG 515063

CHINA

lijian09@mail.ustc.edu.cn

PIOTR OPROCHA

FACUlTy OF APPLIED MATHEMATICS

AGH UNIVERSITY OF SCIENCE AND TECHNOLOGY

AL. MICKIEWICZA 30

30-059 KRAKÓW

POLAND

oprocha@agh.edu.pl

GUOHUA ZHANG

SCHOOL OF MATHEMATICAL SCIENCES AND LMNS

FUDAN UNIVERSITY

HANDAN ROAD No. 220

SHANGHAI 200433

CHINA

and

Shanghai Center for Mathematical Sciences

HANDAN RoAD No. 220

SHANGHAI 200433

CHINA

chiaths.zhang@gmail.com 


\title{
PACIFIC JOURNAL OF MATHEMATICS
}

\author{
msp.org/pjm
}

Founded in 1951 by E. F. Beckenbach (1906-1982) and F. Wolf (1904-1989)

\section{EDITORS}

Don Blasius (Managing Editor)

Department of Mathematics

University of California

Los Angeles, CA 90095-1555

blasius@math.ucla.edu

\author{
Paul Balmer \\ Department of Mathematics \\ University of California \\ Los Angeles, CA 90095-1555 \\ balmer@math.ucla.edu \\ Robert Finn \\ Department of Mathematics \\ Stanford University \\ Stanford, CA 94305-2125 \\ finn@math.stanford.edu \\ Sorin Popa \\ Department of Mathematics \\ University of California \\ Los Angeles, CA 90095-1555 \\ popa@math.ucla.edu
}

\author{
Vyjayanthi Chari \\ Department of Mathematics \\ University of California \\ Riverside, CA 92521-0135 \\ chari@math.ucr.edu \\ Kefeng Liu \\ Department of Mathematics \\ University of California \\ Los Angeles, CA 90095-1555 \\ liu@math.ucla.edu \\ Jie Qing \\ Department of Mathematics \\ University of California \\ Santa Cruz, CA 95064 \\ qing@ cats.ucsc.edu
}

\section{PRODUCTION}

Silvio Levy, Scientific Editor, production@msp.org

\section{SUPPORTING INSTITUTIONS}

ACADEMIA SINICA, TAIPEI

CALIFORNIA INST. OF TECHNOLOGY

INST. DE MATEMÁTICA PURA E APLICADA

KEIO UNIVERSITY

MATH. SCIENCES RESEARCH INSTITUTE

NEW MEXICO STATE UNIV.

OREGON STATE UNIV.

\author{
STANFORD UNIVERSITY \\ UNIV. OF BRITISH COLUMBIA \\ UNIV. OF CALIFORNIA, BERKELEY \\ UNIV. OF CALIFORNIA, DAVIS \\ UNIV. OF CALIFORNIA, LOS ANGELES \\ UNIV. OF CALIFORNIA, RIVERSIDE \\ UNIV. OF CALIFORNIA, SAN DIEGO \\ UNIV. OF CALIF., SANTA BARBARA
}

\author{
Daryl Cooper \\ Department of Mathematics \\ University of California \\ Santa Barbara, CA 93106-3080 \\ cooper@math.ucsb.edu \\ Jiang-Hua Lu \\ Department of Mathematics \\ The University of Hong Kong \\ Pokfulam Rd., Hong Kong \\ jhlu@maths.hku.hk \\ Paul Yang \\ Department of Mathematics \\ Princeton University \\ Princeton NJ 08544-1000 \\ yang@math.princeton.edu
}

These supporting institutions contribute to the cost of publication of this Journal, but they are not owners or publishers and have no responsibility for its contents or policies.

See inside back cover or msp.org/pjm for submission instructions.

The subscription price for 2015 is US \$420/year for the electronic version, and \$570/year for print and electronic.

Subscriptions, requests for back issues and changes of subscribers address should be sent to Pacific Journal of Mathematics, P.O. Box 4163, Berkeley, CA 94704-0163, U.S.A. The Pacific Journal of Mathematics is indexed by Mathematical Reviews, Zentralblatt MATH, PASCAL CNRS Index, Referativnyi Zhurnal, Current Mathematical Publications and Web of Knowledge (Science Citation Index).

The Pacific Journal of Mathematics (ISSN 0030-8730) at the University of California, c/o Department of Mathematics, 798 Evans Hall \#3840, Berkeley, CA 94720-3840, is published twelve times a year. Periodical rate postage paid at Berkeley, CA 94704, and additional mailing offices. POSTMASTER: send address changes to Pacific Journal of Mathematics, P.O. Box 4163, Berkeley, CA 94704-0163.

PJM peer review and production are managed by EditFLOW ${ }^{\circledR}$ from Mathematical Sciences Publishers.

\section{PUBLISHED BY}

\section{mathematical sciences publishers \\ nonprofit scientific publishing}

http://msp.org/

(C) 2015 Mathematical Sciences Publishers 


\section{PACIFIC JOURNAL OF MATHEMATICS}

Volume $277 \quad$ No. $2 \quad$ October 2015

The Borel-Weil theorem for reductive Lie groups

JosÉ ARAUJO and TIM BRATTEN

A curvature flow unifying symplectic curvature flow and pluriclosed

287 flow

SONG DAI

Representations of knot groups into $\mathrm{SL}_{n}(\mathbb{C})$ and twisted Alexander polynomials

Michael HeUsener and JoAn PORTi

Approximations by maximal Cohen-Macaulay modules

HENRIK HOLM

Patterson-Sullivan currents, generic stretching factors and the asymmetric Lipschitz metric for outer space

ILYA KAPOVICH and MARTIN LUSTIG

On recurrence over subsets and weak mixing

JiAn Li, Piotr Oprocha and GUOHUA Zhang

Représentations de Steinberg modulo $p$ pour un groupe réductif sur un 425 corps local

TONY LY

Calculating two-strand jellyfish relations

DAVID PENNEYS and EMILY PETERS 